\title{
PROBABILISTIC INTERVAL TEMPORAL LOGIC AND DURATION CALCULUS WITH INFINITE INTERVALS: COMPLETE PROOF SYSTEMS
}

\author{
DIMITAR P. GUELEV
}

Institute of Mathematics and Informatics, Bulgarian Academy of Sciences e-mail address: gelevdp@math.bas.bg

\begin{abstract}
The paper presents probabilistic extensions of interval temporal logic $(I T L)$ and duration calculus $(D C)$ with infinite intervals and complete Hilbert-style proof systems for them. The completeness results are a strong completeness theorem for the system of probabilistic $I T L$ with respect to an abstract semantics and a relative completeness theorem for the system of probabilistic $D C$ with respect to real-time semantics. The proposed systems subsume probabilistic real-time $D C$ as known from the literature. A correspondence between the proposed systems and a system of probabilistic interval temporal logic with finite intervals and expanding modalities is established too.
\end{abstract}

\section{INTRODUCTION}

The duration calculus $(D C)$ was introduced by Zhou, Hoare and Ravn in [ZHR91] as a logic to specify requirements on real-time systems. $D C$ is a classical predicate intervalbased linear-time logic with one normal binary modality known as chop. $D C$ was originally developed for real time by augmenting the real-time variant of interval temporal logic (ITL, [Mos85, Mos86]) with boolean expressions for state and real-valued terms to denote state durations. $D C$ has been used successfully in many case studies such as [ZZ94, DW96, [SX98, Dan98, LH99]. We refer the reader to [HZ97] or the recent monograph [ZH04] for a comprehensive introduction to $D C$.

Temporal logics such as linear temporal logic $(L T L)$, computation tree logic $(C T L)$ and their timed versions are used mostly as requirements languages for model-checkers such as SMV [McM] and UPPAAL [UPP] which accept descriptions of systems in dedicated input languages. The probabilistic variant of $C T L$ [ASB95] has a similar role in the probabilistic model checker PRISM [KNP01, PRI]. The systems in use are typically propositional, which restricts the variety of properties that can be expressed. This is only in part compensated for by the possibility to do fully algorithmic verification. More complex properties and systems which, e.g., involve unspecified numbers of concurrent processes or unbounded amounts of data have to be viewed as parameterized families and require the development of dedicated techniques. Alternatively, model-checkers are used on instances of the systems

2000 ACM Subject Classification: F.3.1.

Key words and phrases: probabililistic interval temporal logic, duration calculus. 
with artificial bounds on their size, which, however, quickly leads to the notorious state space explosion problem. The use of the logics as reasoning tools and not just as notations is also limited to optimising simplifications such as abstractions. Unlike these systems of logic, the expressive power of $D C$ is geared towards the possibility to capture the semantics of the systems to be verified and therefore it is used as a system description language as well. Examples include the $D C$ semantics of the timed specification language RAISE proposed in [LH99] and the $D C$ semantics of the Verilog hardware specification language [IEE95] proposed in [SX98]. This shifts the interest from the satisfaction of $D C$ formulas by given models towards validity in $D C$.

The needs of applications have brought to life a number of extensions and variants of $D C$. These include state quantifiers and the least fixed point operator [Pan95], alternative sets of interval modalities [Pan96, ZH98, BRZ00, He 99b], enhancements of the semantics to combine real and discrete time [PD98, He 99a, Gue04a and infinite intervals [ZDL95, PWX98, SX98, WX04. The extension of $D C$ by a probability operator replaces the linear model of time of $D C$ by a model based on sets of behaviours with probability on them. Despite the absence of an explicit branching-time modality, the probabilistic $D C(P D C)$ is essentially a branching-time predicate interval-based temporal logic.

$D C$ and, consequently, its extensions are not recursively axiomatisable. The worst case complexity of decision procedures for validity is high even for very restricted subsets of $D C$ such as the so-called propositional $D C$ [ZHS93, Rab98. No interesting quantified decidable subsets of $D C$ seem to be known (The state quantifier in the $\lceil P\rceil$-subset of $D C$ studied in ZHS93 is expressible in that subset and does not increase its ultimate expressive power.) The propositional abstract-time and real-time ITLs with chop are undecidable too. Undecidability is typical of interval-based systems as shown in the early works [HS86] and [Ven91a, Ven91b] where the chop modality was studied as an example of an operator in many-dimensional modal logic. A very simple subset of $D C$ which exhibits its incompleteness was identified in Gue04c]. This is compensated by the convenience of achieving composionality in specification and particularly the specification of sequential composition, which is deemed to be difficult to handle in systems without the chop modality [MO99]. Tool support for ITL and $D C$ has been developed on the basis of PVS [PVS] by combining $I T L$ - and $D C$-specific proof and proof through translation into the higher-order logic input language of PVS [SS94, Hu 99, Ras02]. There is also a model- and validity-checker DCVALID $[\mathrm{Pan}]$, which accepts the discrete time $\lceil P\rceil$-subset of $D C(Q D D C)$ and a combination of $Q D D C$ with $C T L^{*}$ Pan01 and uses MONA Mon as a back-end tool. The expressive power of these subsets of $D C$ is that of weak monadic second order logic with one successor $(W S 1 S)$. DCVALID has been successful in interesting case studies such as that from Pan02. However, the finite-state-based algorithms of MONA impose on it the same ultimate limitations as in other model-checking tools. That is why proof systems are a relatively important instrument for verification by $D C$ and its extensions.

$D C$ was originally introduced for real time, whereas $P D C$ was first introduced in [LRSZ93 for discrete time. A system of real-time $P D C$ was introduced later in [DZ99] where some axioms were proposed too. However, these axioms do not form a complete proof system. Calculation with direct reference to the semantics was used to reason about properties expressed in $P D C$ in both works. More case studies in $P D C$ were given in [Jos95] and recently in [ZH04, which contains a chapter on discrete time $P D C$. The deductive power of the proof system for discrete time $P D C$ used in [ZH04] has not been studied either. 
A first attempt to develop a complete proof system for $P D C$ was made in Gue98, where a system of probabilistic ITL was proposed with the $D C$-specific state expressions with finite variability withdrawn. However, the semantics of that logic had some nonstandard elements for technical reasons, and the proof system was a mixture of ITL and elements from Neighbourhood Logic ( $N L$, [ZH98, RZ97, BRZ00]). Some of these problems were eliminated in [Tri99]. A more streamlined system of probabilistic $N L$ and a complete proof system with respect to its abstract-time semantics was proposed later in Gue00. The use of a (commutative) linearly-ordered group as the model of time in that system after Dutertre's work on abstract-time ITL Dut95a allowed a finitary complete proof system to be obtained. However, $P N L$ still had some loose ends; the questions of the precise correspondence between $P N L$ and the original systems of $P D C$ from [LRSZ93, DZ99] and of the deductive power of the proof system with respect to real-time models remained open. Systems of (non-probabilistic) branching time $N L$ were developed in the recent works [BMS07] and BM05]. Some of these systems can be viewed as the underlying branching time logics of $P N L$. The works [BMS07] and BM05] present the propositional variants of these branching time interval temporal logics and focus on decision procedures for them.

In this paper we first propose another system of probabilistic ITL. Unlike that from Gue98, this system is based on infinite intervals. We propose a proof system for probabilistic ITL with infinite intervals which is complete with respect to the abstract-time semantics based on that for ITL with infinite intervals from [WX04]. The use of infinite intervals removes the need to admix $N L$ modalities in proofs, which was done in Gue98. Then we develop a system of probabilistic $D C(P D C)$ as an extension of the proposed probabilistic $I T L$ and demonstrate that adding the $D C$ axioms and rules known from [HZ92] to our proof system for this probabilistic ITL leads to a proof system for $P D C$ with is complete with respect to real-time models relative to validity at the real-time-based frame in probabilistic $I T L$ with infinite intervals. The incompleteness of $D C$ implies that relative completeness like that from [HZ92 for basic $D C$ is the best we can have with a finitary proof system. Finally, we describe satisfaction-preserving translations between $N L$-based $P D C$ and the system of $P D C$ with infinite intervals that we propose.

Our system of $P D C$ has some slight enhancements in comparison with the original probabilistic $D C$ from LRSZ93, DZ99. They both improve its expressivity and facilitate the design of the proof system. The first enhancement is a simplification. We remove the extra reference time point needed to define the probability operator. The role of this time point is naturally transferred to the flexible constant $\ell$ which expresses interval lengths in $D C$. This extends the possibilities for meaningful nesting of occurrences of the probability operator and allows the expression of probabilities of properties which are probabilistic themselves. The second enhancement is the use of infinite intervals. It is a consequence of our developing of $P D C$ as an extension of an infinite-interval-based system of probabilistic ITL. As mentioned above, this makes it possible to avoid the use of an expanding modality such as those of $N L$, which was made in Gue00. The combination of the chop modality and infinite intervals has the expressive power of expanding modalities with the advantage of keeping the introspectivity of chop, which is a technically useful property. We discuss the trade-offs between $N L$ and ITL in Section 9. The last enhancement is the replacement of the probabilistic timed automata which were used in [DZ99] to define sets of behaviours and the respective probability functions for $P D C$ models by arbitrary systems of probability functions, which can be constrained by additional axioms in $P D C$ theories. One such constraint that we study in detail is the requirement on all the probability functions in a 
model to be consistent with a global probability function which is defined on the space of all the behaviours of the modelled system. Models which describe the behaviour of automata like those involved in the definition of the original system of real-time $D C$ from [DZ99] can be described by $P D C$ theories in this more general setting too.

Structure of the paper. After the necessary preliminaries on ITL with infinite intervals and $D C$ we introduce our system of probabilistic ITL with infinite intervals and a proof system for it. We prove the completeness of this proof system with respect to the abstract semantics of probabilistic ITL, which is the main result of the paper. Then we propose axioms which constrain the system of probability functions in models of PITL to be consistent with a global probability function to the extent that this constraint can be formulated in the setting of abstract probabilies. In the rest of the paper we introduce a system of probabilistic $D C$ as an extension of the new system of probabilistic ITL by state expressions and duration terms for them based on the real-time frame of probabilistic ITL. We show how this system of $P D C$ subsumes the system proposed in [DZ99]. The main result about $P D C$ is the completeness of the well-known axioms of $D C$ from HZ92 relative to validity in real-time and -probability-based models for probabilistic ITL. Before concluding the paper we explain the correspondence between $P N L$ from Gue00 and the infinite-interval based PITL proposed in this paper. We conclude by explaining some of the limitations of the scope of its main results.

\section{Preliminaries}

In this section we give preliminaries on $I T L$ and $D C$ with infinite intervals as known from [ZDL95, PWX98, SX98, WX04 and the probability operator of PDC as introduced in [LRSZ93, DZ99].

1.1. Interval temporal logic with infinite intervals. Here follows a brief formal introduction to ITL with infinite intervals as presented in [WX04, which extends the finite interval abstract-time system of ITL proposed and studied in [Dut95a].

1.1.1. Language. An ITL vocabulary consists of constant symbols $c, d, \ldots$, individual variables $x, y, z, \ldots$, function symbols $f, g, \ldots$ and relation symbols $R, \ldots$ Constant, function and relation symbols can be either rigid or flexible. Below it becomes clear that rigid symbols have the same meaning at all times, whereas the meaning of flexible symbols can depend on the reference time interval. The rigid constants 0 and $\infty$, addition + , equality $=$, the flexible constant $\ell$, which always evaluates to the length of the reference interval, and a countably infinite set of individual variables are mandatory in every $I T L$ vocabulary. We denote the arity of function and relation symbols $s$ by $\# s$.

Given a vocabulary, the definition of an ITL language is essentially that of its sets of terms $t$ and formulas $\varphi$, which can be defined by the following BNFs:

$$
\begin{aligned}
& t::=c|x| f(t, \ldots, t) \\
& \varphi \quad:=\perp|R(t, \ldots, t)|(\varphi \Rightarrow \varphi)|(\varphi ; \varphi)| \exists x \varphi
\end{aligned}
$$

Many authors use the alternative notation $\varphi-\psi$ for formulas $(\varphi ; \psi)$ which are built with the chop modality. 
Terms and formulas with no occurrences of flexible symbols are called rigid. Other terms and formulas are called flexible. The set of the variables which have free occurrences in a formula $\varphi$ is denoted by $F V(\varphi)$.

1.1.2. Models and satisfaction. A finite interval $I T L$ frame consists of a linearly ordered set $\langle T, \leq\rangle$ called the time domain, a monoid $\langle D, 0,+\rangle$ called the duration domain and a function $m: \mathbf{I}(T) \rightarrow D$ called the measure function, where

$$
\mathbf{I}(T)=\left\{\left[\tau_{1}, \tau_{2}\right]: \tau_{1}, \tau_{2} \in T, \tau_{1} \leq \tau_{2}\right\}
$$

is the set of the closed and bounded intervals in $T$. The monoid $\langle D, 0,+\rangle$ is required to satisfy some additional axioms. The full list of axioms is:

$(D 1) \quad x+(y+z)=(x+y)+z$

(D2) $\quad x+0=0+x=x$

(D3) $x+y=x+z \Rightarrow y=z, x+z=y+z \Rightarrow x=y$

(D4) $x+y=0 \Rightarrow x=y=0$

(D5) $\exists z(x+z=y \vee y+z=x), \exists z(z+x=y \vee z+y=x)$

The measure function $m$ is required to satisfy the axioms:

(M1) $m\left(\left[\tau_{1}, \tau_{2}\right]\right)=m\left(\left[\tau_{1}, \tau_{2}^{\prime}\right]\right) \Rightarrow \tau_{2}=\tau_{2}^{\prime}$

(M2) $m\left(\left[\tau_{1}, \tau\right]\right)+m\left(\left[\tau, \tau_{2}\right]\right)=m\left(\left[\tau_{1}, \tau_{2}\right]\right)$

(M3) $m\left(\left[\tau_{1}, \tau_{2}\right]\right)=x+y \Rightarrow \exists \tau\left(m\left(\left[\tau_{1}, \tau\right]\right)=x\right)$

In the case of $I T L$ with infinite intervals the time domain $\langle T, \leq\rangle$ is supposed to have a distinguished greatest element $\infty$ and $m$ is defined on the set $\tilde{\mathbf{I}}(T)=\mathbf{I}^{f i n}(T) \cup \mathbf{I}^{i n f}(T)$, where

$$
\mathbf{I}^{\text {fin }}(T)=\left\{\left[\tau_{1}, \tau_{2}\right]: \tau_{1}, \tau_{2} \in T, \tau_{1} \leq \tau_{2}<\infty\right\} \text { and } \mathbf{I}^{\text {inf }}(T)=\{[\tau, \infty]: \tau \in T, \tau<\infty\} .
$$

The duration domain is augmented with a greatest element $\infty$ too. The axiom D3 is weakened to

$\left(D 3^{\prime}\right) \quad x+y=x+z \Rightarrow x=\infty \vee y=z, x+z=y+z \Rightarrow z=\infty \vee x=y$

and the following axioms about durations and the measure functions are added:

(D6) $\quad x+y=\infty \Leftrightarrow x=\infty \vee y=\infty$

(M4) $m\left(\left[\tau_{1}, \tau_{2}\right]\right)=\infty$ iff $\tau_{2}=\infty$

Given $\sigma_{1}, \sigma_{2} \in \tilde{\mathbf{I}}(T)$ such that $\max \sigma_{1}=\min \sigma_{2}$, we denote $\sigma_{1} \cup \sigma_{2}$ by $\sigma_{1} ; \sigma_{2}$.

A function $I$ on an $I T L$ vocabulary $\mathbf{L}$ is an interpretation of $\mathbf{L}$ into a frame

$F=\langle\langle T, \leq, \infty\rangle,\langle D,+, 0, \infty\rangle, m\rangle$ if it satisfies the conditions:

$I(c), I(x) \in D$ for rigid constants $c$ and individual variables $x$;

$I(f) \in\left(D^{\# f} \rightarrow D\right)$ for rigid function symbols $f$;

$I(R) \in\left(D^{\# R} \rightarrow\{0,1\}\right)$ for rigid relation symbols $R$;

$I(c) \in(\tilde{\mathbf{I}}(T) \rightarrow D), I(f) \in\left(\tilde{\mathbf{I}}(T) \times D^{\# f} \rightarrow D\right), I(R) \in\left(\tilde{\mathbf{I}}(T) \times D^{\# R} \rightarrow\{0,1\}\right)$ for flexible $c, f$ and $R$;

$I(0)=0, I(\infty)=\infty, I(+)=+, I(=)$ is $=$ and $I(\ell)=m$.

An infinite-interval model for an $I T L$ vocabulary $\mathbf{L}$ is a pair of the form $\langle F, I\rangle$ such that $F$ is a frame and $I$ is an interpretation of $\mathbf{L}$ into $F$.

Definition 1.1. Given a model $\langle F, I\rangle$, the values $I_{\sigma}(t)$ of terms $t$ at intervals $\sigma \in \tilde{\mathbf{I}}(T)$ is defined by the clauses: 


$$
\begin{array}{ll}
I_{\sigma}(x) & =I(x) \text { for individual variables } x \\
I_{\sigma}(c) & =I(c) \text { for rigid constants } c \\
I_{\sigma}\left(f\left(t_{1}, \ldots, t_{\# f}\right)\right) & =I(f)\left(I_{\sigma}\left(t_{1}\right), \ldots, I_{\sigma}\left(t_{\# f}\right)\right) \text { for rigid function symbols } f \\
I_{\sigma}(c) & =I(c)(\sigma) \text { for flexible } c \\
I_{\sigma}\left(f\left(t_{1}, \ldots, t_{\# f}\right)\right) & =I(f)\left(\sigma, I_{\sigma}\left(t_{1}\right), \ldots, I_{\sigma}\left(t_{\# f}\right)\right) \text { for flexible } f
\end{array}
$$

In particular, $I_{\sigma}(\ell)=m(\sigma)$, which means that the function on $\tilde{\mathbf{I}}$ which is the meaning of the flexible constant $\ell$ always evaluates to the length of the reference interval $\sigma$.

Definition 1.2. Let $I$ be an interpretation of some $I T L$ vocabulary $\mathbf{L}$ into a frame $F$ whose duration domain is $\langle D,+, 0, \infty\rangle$. Let $x$ be an individual variable in $\mathbf{L}$ and $d \in D$. Then the interpretation $J$ of $\mathbf{L}$ into $F$ which is defined by the equalities

$$
J(x)=d \text { and } J(s)=I(s) \text { for } s \in \mathbf{L} \backslash\{x\}
$$

is denoted by $I_{x}^{d}$ and is called a $x$-variant of $I$. We abbreviate $\left(\ldots\left(I_{x_{1}}^{d_{1}}\right)_{x_{2}}^{d_{2}} \ldots\right)_{x_{n}}^{d_{n}}$ by $I_{x_{1}, \ldots, x_{n}}^{d_{1}, \ldots, d_{n}}$ and call it an $x_{1}, \ldots, x_{n}$-variant of $I$. An $x_{1}, \ldots, x_{n}$-variant of $I$ for some finite list of variables $x_{1}, \ldots, x_{n}$ is called just variant.

The modelling relation $\models$ on models based on some frame $F$, intervals $\sigma$ and formulas in the vocabulary $\mathbf{L}$ is defined by the clauses:

$$
\begin{array}{ll}
\langle F, I\rangle, \sigma \not \models \perp & \\
\langle F, I\rangle, \sigma \models R\left(t_{1}, \ldots, t_{n}\right) & \text { iff } I(R)\left(I_{\sigma}\left(t_{1}\right), \ldots, I_{\sigma}\left(t_{n}\right)\right)=1 \text { for rigid } R \\
\langle F, I\rangle, \sigma \models R\left(t_{1}, \ldots, t_{n}\right) & \text { iff } I(R)\left(\sigma, I_{\sigma}\left(t_{1}\right), \ldots, I_{\sigma}\left(t_{n}\right)\right)=1 \text { for flexible } R \\
\langle F, I\rangle, \sigma \models(\varphi \Rightarrow \psi) & \text { iff either }\langle F, I\rangle, \sigma \not \varphi \text { or }\langle F, I\rangle, \sigma \models \psi \\
\langle F, I\rangle, \sigma \models(\varphi ; \psi) & \text { iff } \\
& \langle F, I\rangle, \sigma_{1} \models \varphi \text { and }\langle F, I\rangle, \sigma_{2} \models \psi \\
& \text { for some } \sigma_{1} \in \mathbf{I} \mathbf{I}^{f i n}\left(T_{F}\right) \text { and } \sigma_{2} \in \tilde{\mathbf{I}}\left(T_{F}\right) \text { such that } \sigma_{1} ; \sigma_{2}=\sigma \\
\langle F, I\rangle, \sigma \models \exists x \varphi & \text { iff }\left\langle F, I_{x}^{d}\right\rangle, \sigma \models \varphi \text { for some } d \in D
\end{array}
$$

1.1.3. Abbreviations and precedence of operators. The binary relation symbol $\leq$ is defined in $I T L$ by the equivalence

$$
x \leq y \Leftrightarrow \exists z(x+z=y) .
$$

The customary infix notation for,$+ \leq$ and $=$ is used in ITL. $\top, \wedge, \Rightarrow$ and $\Leftrightarrow, \forall, \neq, \geq,<$ and $>$ are used in the usual way. We denote the universal closure $\forall x_{1} \ldots \forall x_{n} \varphi$ of a formula $\varphi$ where $\left\{x_{1}, \ldots, x_{n}\right\}=F V(\varphi)$ by $\forall \varphi$.

Since (.; .) is associative, we omit parentheses in formulas with consecutive occurrences of (.;.). Here follow the infinite-interval versions of some $I T L$ abbreviations:

$\diamond \varphi \rightleftharpoons(\top ; \varphi ; \top) \vee(\top ; \varphi), \square \varphi \rightleftharpoons \neg \diamond \neg \varphi$.

Note that $\square$ and $\diamond$ abbreviate different constructs in the original discrete-time system of $I T L$ of Moszkowski. Our usage originates from the literature on $D C$. The disjunctive member $(T ; \varphi)$ in the definition of $\diamond$ is relevant only at infinite intervals. The formula $(\top ; \varphi ; \top)$ without it restricts the subinterval which satisfies $\varphi$ to be finite.

We assume that $\diamond$ and $\square$ bind more tightly and (.;.) binds less tightly than the boolean connectives. 
1.1.4. Proof system. A complete proof system for abstract-time $I T L$ with finite intervals is given in [Dut95a. The following axioms and rules have been shown to form a complete proof system for ITL with infinite intervals when added to a Hilbert-style proof system for classical first-order predicate logic and the axioms $D 1, D 2, D 3^{\prime}, D 4-D 6$ about durations in WX04]:

$$
\begin{array}{ll}
(A 1) & (\varphi ; \psi) \wedge \neg(\chi ; \psi) \Rightarrow(\varphi \wedge \neg \chi ; \psi),(\varphi ; \psi) \wedge \neg(\varphi ; \chi) \Rightarrow(\varphi ; \psi \wedge \neg \chi) \\
(A 2) & ((\varphi ; \psi) ; \chi) \Leftrightarrow(\varphi ;(\psi ; \chi)) \\
(R) & (\varphi ; \psi) \Rightarrow \varphi,(\psi ; \varphi) \Rightarrow \varphi \text { if } \varphi \text { is rigid } \\
(B) & (\exists x \varphi ; \psi) \Rightarrow \exists x(\varphi ; \psi),(\psi ; \exists x \varphi) \Rightarrow \exists x(\psi ; \varphi) \text { if } x \notin F V(\psi) \\
(L 1) & (\ell=x ; \varphi) \Rightarrow \neg(\ell=x ; \neg \varphi),(\varphi ; \ell=x \wedge x \neq \infty) \Rightarrow \neg(\neg \varphi ; \ell=x) \\
(L 2) & \ell=x+y \wedge x \neq \infty \Leftrightarrow(\ell=x ; \ell=y) \\
(L 3) & \varphi \Rightarrow(\ell=0 ; \varphi), \varphi \wedge \ell \neq \infty \Rightarrow(\varphi ; \ell=0) \\
(S 1) & (\ell=x \wedge \varphi ; \psi) \Rightarrow \neg(\ell=x \wedge \neg \varphi ; \chi) \\
(P 1) & \neg(\ell=\infty ; \varphi) \\
(P 2) & (\varphi ; \ell=\infty) \Rightarrow \ell=\infty \\
(P 3) & (\varphi ; \ell \neq \infty) \Rightarrow \ell \neq \infty \\
(N) & \frac{\varphi}{\neg(\neg \varphi ; \psi)}, \neg \frac{\varphi}{\neg(\psi ; \neg \varphi)} \\
& \frac{\varphi \Rightarrow \psi}{(\text { Mono })} \\
& \frac{\varphi ; \chi) \Rightarrow(\psi ; \chi)}{(\varphi)} \frac{\varphi \Rightarrow \psi}{(\chi ; \varphi) \Rightarrow(\chi ; \psi)}
\end{array}
$$

The presence of the modality (.;.) and flexible symbols in ITL brings a restriction on the use of first order logic axioms which involve substitution such as

$$
\left(\exists_{r}\right)[t / x] \varphi \Rightarrow \exists x \varphi .
$$

The application of this axiom is correct only if no variable in $t$ becomes bound due to the substitution, and either $t$ is rigid or (.;.) does not occur in $\varphi$, because the value of a flexible term could be different at the different intervals which are involved in evaluating formulas with $(. ;$.$) .$

The correctness of the proof system can be established by a direct check. Here follow some comments and informal reading of the axioms and the proof rules which can be helpful for their understanding too. A1 states that if chopping into a $\varphi$-subinterval and a $\psi$-subinterval is possible, but chopping into a $\chi$-subinterval and a $\psi$-subinterval is not, then any chopping into a $\varphi$ - and a $\psi$-subinterval would lead to a $\varphi$-subinterval which additionally satisfies the negation of $\chi$. In the presence of the rules Mono and propositional tautologies one can choose between $A 1$ and the axiom

$$
(\alpha ; \psi) \vee(\beta ; \psi) \Leftrightarrow(\alpha \vee \beta ; \psi),
$$

which can be described as distributivity of (.;.) over $\vee$. Axiom $B$ can be viewed as an parametric analogon of this distributivity axiom, with $\exists x$ to be read as parametric (possibly infinitary) disjunction. $A 2$ is just the associativity of (.;.). $R$ states that the satisfaction of rigid formulas does not depend on the reference interval. $L 1$ and $S 1$ express that if, upon dividing an interval, the duration of one of the subintervals is fixed, then the properties of both subintervals are completely determined. This is so because the subintervals themselves are uniquely determined. $L 2$ is the additivity of length. $P 2$ and $P 3$ give separate treatment to some special cases of additivity that arise from the presence of infinitely long intervals. 
L3 states that intervals of length 0 can be assumed at either end of any interval. P3 rules out the interval $[\infty ; \infty]$. The rules $N$ state that valid formulas are valid in subintervals too. These rules are the standard form of the modal logic rule $\varphi / \square \varphi$, yet about the binary modality (.; .). The fact that weakening the condition on a subinterval in a (.;.)-formula can only facilitate the satisfiability of the whole (.;.)-formula is expressed by the rules Mono.

1.2. $D C$ with infinite intervals. The formal definition of $D C$ with infinite intervals as an extension of the logic of the real-time-based frame of $I T L$ with infinite intervals below is after [ZDL95]. The main feature of $D C$ relative to $I T L$ are state expressions which are propositional formulas that denote piece-wise constant $\{0,1\}$-valued functions of time. Unlike purely-ITL flexible symbols, $D C$ state expressions denote functions on time points and not intervals.

1.2.1. Language. $D C$ vocabularies are $I T L$ vocabularies extended by state variables $P, Q, \ldots$ State variables are used to build state expressions $S$ which have the syntax

$$
S \quad::=\mathbf{0}|P| S \Rightarrow S
$$

and in turn appear as the argument of duration terms $\int S$ which are the $D C$-specific construct in the syntax of terms $t$ :

$$
t::=c|x| v\left|\int S\right| f(t, \ldots, t)
$$

Duration terms are regarded as flexible. The syntax of formulas is as in ITL.

Flexible constants and 0-ary flexible predicate letters in $D C$ are also known as temporal variables and temporal propositional letters, respectively.

1.2.2. Semantics. We are only interested in real-time $D C$ which is based on the $I T L$ frame

$$
F_{\mathbf{R}}=\left\langle\langle\overline{\mathbf{R}}, \leq, \infty\rangle,\left\langle\overline{\mathbf{R}}_{+},+, 0, \infty\right\rangle, \lambda \sigma \cdot \max \sigma-\min \sigma\right\rangle
$$

where $\overline{\mathbf{R}}=\mathbf{R} \cup\{\infty\}$ and $\overline{\mathbf{R}}_{+}=\{x \in \overline{\mathbf{R}}: x \geq 0\}$.

$D C$ interpretations extend $I T L$ interpretations to provide values for state variables, which are functions of type $\mathbf{R} \rightarrow\{0,1\}$ that satisfy the following finite variability requirement:

For every pair $\tau_{1}, \tau_{2} \in \mathbf{R}$ such that $\tau_{1}<\tau_{2}$, and every state variable $P$ there exist an $n<\omega$ and $\tau_{1}^{\prime}, \ldots, \tau_{n}^{\prime} \in \mathbf{R}$ such that $\tau_{1}=\tau_{1}^{\prime}<\ldots<\tau_{n}^{\prime}=\tau_{2}$ and $I(P)$ is constant on the semi-open intervals $\left[\tau_{i}^{\prime}, \tau_{i+1}^{\prime}\right), i=1, \ldots, n-1$.

Given an interpretation $I$, the values $I_{\tau}(S)$ of state expressions $S$ at time $\tau \in \mathbf{R}$ are defined by the equalities

$$
\begin{array}{ll}
I_{\tau}(\mathbf{0}) & =0 \\
I_{\tau}(P) & =I(P)(\tau) \text { for state variables } P \\
I_{\tau}\left(S_{1} \Rightarrow S_{2}\right) & =\max \left(1-I_{\tau}\left(S_{1}\right), I_{\tau}\left(S_{2}\right)\right)
\end{array}
$$

The value $I_{\sigma}\left(\int S\right)$ of duration term $\int S$ at interval $\sigma \in \tilde{\mathbf{I}}(\overline{\mathbf{R}})$ is defined by the equality

$$
I_{\sigma}\left(\int S\right)=\int_{\min \sigma}^{\max \sigma} I_{\tau}(S) d \tau
$$

Note that $I_{\sigma}\left(\int S\right)$ can be $\infty$ for $\sigma \in \mathbf{I}^{\text {inf }}(\overline{\mathbf{R}})$. The values of other kinds of terms and $\models$ are defined as in ITL. 
1.2.3. Abbreviations. The boolean connectives $\neg, \vee, \wedge$ and $\Leftrightarrow$ are used in state expressions as abbreviations in the usual way. The following abbreviations are specific to $D C$ :

$$
\begin{aligned}
& \mathbf{1} \rightleftharpoons \neg \mathbf{0} \\
& \llbracket S \rrbracket \rightleftharpoons \int S=\ell \wedge \ell \neq 0
\end{aligned}
$$

Sometimes $\ell$ is introduced as an abbreviation for $\int \mathbf{1}$.

1.2.4. Proof system. The axioms and rules below were proposed in [HZ92] for DC with finite intervals.
$(D C 1) \quad \int \mathbf{0}=0$
$(D C 2) \quad \int \mathbf{1}=\ell$
(DC3) $\int S \geq 0$
$(D C 4) \quad \int S_{1}+\int S_{2}=\int\left(S_{1} \vee S_{2}\right)+\int\left(S_{1} \wedge S_{2}\right)$
$(D C 5) \quad\left(\int S=x ; \int S=y\right) \Rightarrow \int S=x+y$
(DC6) $\int S_{1}=\int S_{2}$ if $S_{1}$ and $S_{2}$ are propositionally equivalent
(IR1) $\quad \frac{[\ell=0 / A] \varphi \varphi \Rightarrow[A \vee(A ; \llbracket S \rrbracket \vee \llbracket \neg S \rrbracket) / A] \varphi}{[\top / A] \varphi}$
$(I R 2) \quad \frac{[\ell=0 / A] \varphi \varphi \Rightarrow[A \vee(\llbracket S \rrbracket \vee \llbracket \neg S \rrbracket ; A) / A] \varphi}{[\top / A] \varphi}$

These axioms and rules have been shown to be complete with respect to the finiteinterval variant $\left\langle\langle\mathbf{R}, \leq\rangle,\left\langle\mathbf{R}_{+},+, 0\right\rangle, \lambda \sigma \cdot \max \sigma-\min \sigma\right\rangle$ of $F_{\mathbf{R}}$ relative to validity in the class of the ITL models which are based on the finite-interval variant of $F_{\mathbf{R}}$ in [HZ92].

The correctness of $I R 1$ and $I R 2$ is based on the finite variability of state. Since every finite interval can be partitioned into finitely many subintervals in which the state expression $S$ is constant, proving the validity of a property $\varphi$ about zero-length intervals and proving that the validity of $\varphi$ at intervals with $n$ alternations of the value of $S$ implies the validity of the same property about intervals with $n+1$ such alternations is sufficient to conclude that $\varphi$ holds about intervals with any finite number of alternations of the value of $S$. This, by the assumption of finite variability, means that $\varphi$ is valid about all intervals. The completeness proof from [HZ92 involves two theorems which can be derived using the rules $I R 1$ and $I R 2$, instead of the rules themselves. The second of these theorems does not hold for infinite intervals and therefore we modify it appropriately:

$(T 1) \quad \ell=0 \vee(\| S \rrbracket ; \top) \vee(\llbracket \neg S \rrbracket ; \top)$

$(T 2) \quad \ell=0 \vee \ell=\infty \vee(\top ; \llbracket S \rrbracket) \vee(\top ; \llbracket \neg S \rrbracket)$

The use of $T 1$ and $T 2$ instead of $I R 1$ and $I R 2$ brings technical convenience to the representation of $D C$ as a theory in ITL with $D C 1-D C 6, T 1$ and $T 2$ as its axioms in the proof of relative completeness.

We take $D C 1-D C 6, T 1$ and the infinite-interval version of $T 2$ as axioms to form a relatively complete proof system for $D C$ with infinite intervals and disregard the rules $I R 1$ and $I R 2$ in the rest of the paper. The proof of the relative completeness of this system follows closely the pattern of the original proof from [HZ92]. It appears as part of the proof of the relative completeness of our infinite-interval-based system of probabilistic $D C$ in Section 8 .

1.3. Probabilistic $D C$ for real time. Probabilistic $D C$ was first introduced for discrete time in LRSZ93]. There is a chapter on discrete time probabilistic DC in ZH04 too. Here follows the formal definition of real-time probilistic $D C$ as introduced in [DZ99]. 
1.3.1. Real-time probabilistic automata. The semantics of the real-time probabilistic $D C$ as originally proposed in [DZ99] is based on a class of real-time probabilistic automata.

Definition 1.3. A finite probabilistic timed automaton is a system of the form

$$
\mathbf{A}=\left\langle S, A, s_{0},\left\langle q_{a}, a \in A\right\rangle,\left\langle p_{a}: a \in A\right\rangle\right\rangle
$$

where:

$S$ is a finite set of states;

$A \subset\left\{\left\langle s, s^{\prime}\right\rangle: s, s^{\prime} \in S, s \neq s^{\prime}\right\}$ is a set of transitions;

$s_{0} \in S$ is called the initial state;

$q_{a} \in[0,1]$ is the choice probability for transition $a \in A$;

$p_{a} \in\left(\mathbf{R}_{+} \rightarrow \mathbf{R}_{+}\right)$is the duration probability density of transition $a$.

Given the automaton $\mathbf{A}, A_{s}$ denotes $\left\{s^{\prime} \in S:\left\langle s, s^{\prime}\right\rangle \in A\right\}$. If $a \in A$ and $a=\left\langle s, s^{\prime}\right\rangle$, then $s$ and $s^{\prime}$ are denoted by $a^{-}$and $a^{+}$, respectively. Choice probabilities $q_{a}$ are required to satisfy $\sum_{a \in A_{s}} q_{a}=1$ for $A_{s} \neq \emptyset$. Probability densities $p_{a}$ are required to satisfy $\int_{0}^{\infty} p_{a}(\tau) d \tau=1$.

An automaton $\mathbf{A}$ of the form (1.2) works by going through a finite or infinite sequence of states $s_{0}, s_{1}, \ldots, s_{n}, \ldots$ such that $\left\langle s_{i}, s_{i+1}\right\rangle \in A$ for all $i$. Each transition has a duration $d_{i}$, which is the time that elapses before $s_{i}$ changes to $s_{i+1}$. Thus individual behaviours of A can be represented as sequences of the form

$$
\left\langle a_{0}, d_{0}\right\rangle, \ldots,\left\langle a_{n}, d_{n}\right\rangle, \ldots
$$

where $a_{i} \in A, d_{i} \in \mathbf{R}_{+}, a_{0}^{-}=s_{0}$ and $a_{i}^{+}=a_{i+1}^{-}$for all $i$. Having arrived at state $s, \mathbf{A}$ chooses transition $a \in A_{s}$ with probability $q_{a}$. The probability for the duration of $a$ to be in $\left[\tau_{1}, \tau_{2}\right]$ is $\int_{\tau_{1}}^{\tau_{2}} p_{a}(\tau) d \tau$.

Automata of the above type are closely related to the probabilistic real-time processes known from [ACD91, ACD92].

1.3.2. DC models for real-time probabilistic automata behaviours. Probabilistic DC was introduced in [DZ99] for vocabularies built to describe the behaviours of given real-time probabilistic automata. The $D C$ vocabulary $\mathbf{L}_{\mathbf{A}}$ for (1.2) has the states $s \in S$ as its state variables. The only other non-logical symbols are the mandatory ones. A $D C$ interpretation of $\mathbf{L}_{\mathbf{A}}$ describes the behaviour (1.3) of $\mathbf{A}$ if for all $i<\omega \tau \in\left[\sum_{j<i} d_{j}, \sum_{j \leq i} d_{j}\right)$ implies that $I_{\tau}\left(s_{k}\right)=1$ just for $k=i$.

1.3.3. Satisfaction probability of $D C$ formulas and probabilistic $D C$ for real time. Given a real-time probabilistic automaton (1.2), the set $\mathbf{W}_{\mathbf{A}}$ of all the interpretations of $\mathbf{L}_{\mathbf{A}}$ which describe possible behaviours of $\mathbf{A}$ can be endowed with a probability function $\mu_{\mathbf{A}}$. Given $A \subseteq \mathbf{W}_{\mathbf{A}}, \mu_{\mathbf{A}}(A)$ can be defined as the probability for $\mathbf{A}$ to have a behaviour described by an interpretation in $A$. The sets $A$ in the domain of $\mu_{\mathbf{A}}$ should be chosen from some appropriate boolean algebra of subsets of $2^{\mathbf{W}_{\mathbf{A}}}$. Details on the definition of $\mu_{\mathbf{A}}$, including explicit formulas for $\mu_{\mathbf{A}}$ in terms of $p_{a}$ and $q_{a}$, can be found in [DZ99].

Given $\tau \in \mathbf{R}_{+}$and a $D C$ formula $\varphi$ in the vocabulary $\mathbf{L}_{\mathbf{A}}$, the value of the $P D C$ term $\mu_{\mathbf{A}}(\varphi)(\tau)$ is defined as

$$
\mu_{\mathbf{A}}\left(\left\{I \in \mathbf{W}_{\mathbf{A}}: I,[0, \tau] \models \varphi\right\}\right) .
$$


Probabilistic $D C$ for real time was introduced in DZ99] by enhancing $D C$ with terms of the form $\mu(\varphi)(t)$ where $\varphi$ is a $D C$ formula in $\mathbf{L}_{\mathbf{A}}$ for some automaton $\mathbf{A}$ and $t$ is a term. The values of such terms were defined by the equality

$$
I_{\sigma}(\mu(\varphi)(t))=\mu_{\mathbf{A}}(\varphi)\left(I_{\sigma}(t)\right)
$$

Note that $I_{\sigma}(\mu(\varphi)(t))$ depends on $\sigma$ only through the value of $t$. This means that $\mu(\varphi)(t)$ is rigid iff $t$ is.

\section{Probabilistic ITL With infinite intervals}

In this section we extend abstract-time $I T L$ with infinite intervals by a probability operator which generalises the operator $\mu().($.$) of P D C$ from [LRSZ93, DZ99]. The new probability operator is more expressive and syntactically simpler than $\mu().($.$) . Instead of$ the binary $\mu(\varphi)(t)$ we use a unary $p(\varphi)$ which takes the formula argument $\varphi$ of $\mu$. The semantics of $p(\varphi)$ given below makes it clear that the term argument $t$ which determines the length of the interval at which $\varphi$ is to be evaluated need not be written separately because $\mu(\varphi)(t)$ can be expressed as $p((\varphi \wedge \ell=t ; \top))$. To accomodate the arithmetics of probabilities, abstract-time frames for the new system of probabilistic ITL include a similarly abstract probability domain. We use the acronym PITL for the new system. PITL and its proof system is the main topic of this paper. As it becomes clear below, PITL can be extended to $P D C$ in a straightforward way.

2.1. Language. PITL vocabularies are two-sorted, with durations and probabilities being the two sorts. For this reason, instead of just arities, the non-logical symbols have types which determine the sorts of each argument in the cases of function and relation symbols, and the sort of terms built using the symbol for constants, variables and function symbols. A term or atomic formula $s\left(t_{1}, \ldots, t_{\# s}\right)$ is well formed only if the sorts of the argument terms $t_{1}, \ldots, t_{\# s}$ match the type of $s$.

Along with the mandatory non-logical symbols $0, \infty,+$ and $\ell$ of the duration sort, PITL vocabularies are required to include the rigid constants 0 and 1 and addition + of the probability sort. Equality = is included for each sort too. We use the same characters to denote these otherwise distinct symbols as long as this causes no confusion. We assume countably infinite sets of individual variables of either sort and no more than countablyinfinite sets of other symbols in PITL vocabularies.

The syntax of PITL terms extends that from ITL by terms of the form $p(\varphi)$ where $\varphi$ is a formula. These terms are of the probability sort and we call them probability terms. $F V(p(\varphi))=F V(\varphi)$ and $p(\varphi)$ is rigid iff $\varphi$ is rigid.

The syntax of formulas is as in ITL.

2.2. Models and satisfaction. The main part of a PITL model is a collection of interpretations of the given vocabulary into a given two-sorted frame for ITL with infinite intervals. These interpretations are meant to describe the possible behaviours of a modelled system. Unlike the original $P D C$ models, which assume a global probability function that is derived from the laws of probabilistic behaviour of appropriate automata, we assume a probability distribution to model the probabilistic branching of every behaviour at every time point. Restrictions on the system of probability distributions which, e.g., force them to model the 
choice and duration probabilities of an appropriate automaton can be imposed by additional axioms such as those from Section 6.3.

Definition 2.1. A PITL frame is a tuple of the form

$$
F=\langle\langle T, \leq, \infty\rangle,\langle D,+, 0, \infty\rangle,\langle U,+, 0,1\rangle, m\rangle,
$$

where $\langle T, \leq, \infty\rangle,\langle D,+, 0, \infty\rangle$ and $m$ are as in frames for $I T L$ with infinite intervals and $\langle U,+, 0,1\rangle$ is a commutative monoid with the additional constant 1 , which is called the probability domain. $\langle U,+, 0,1\rangle$ is supposed to satisfy some additional axioms. Here follows the full list:

$$
\begin{array}{ll}
(U 1) & x+(y+z)=(x+y)+z \\
(U 2) & x+y=y+x \\
(U 3) & x+0=x \\
(U 4) & x+y=x+z \Rightarrow y=z \\
(U 5) & x+y=0 \Rightarrow x=y=0 \\
(U 6) & \exists z(x+z=y \vee y+z=x) \\
(U 7) & 0 \neq 1
\end{array}
$$

We use the same symbols for + and 0 in both duration domains and probability domains, despite that they are different entities, as long as this causes no confusion. Probability domains are assumed to be ordered by the relation $\leq$ which is defined by (1.1) like in the case of durations.

For the rest of the section $\mathbf{L}$ denotes some PITL vocabulary and $F$ is some PITL frame with its components named as above.

Definition 2.2. A PITL interpretation of $\mathbf{L}$ into $F$ is a function $I$ on $\mathbf{L}$ which satisfies the conditions:

$I(c), I(x) \in A$ for rigid constants $c$ and individual variables $x$ where $A$ is either $D$ or $U$, depending on the sort of the symbol;

$I(f) \in\left(A_{1} \times \ldots \times A_{\# f} \rightarrow A_{\# f+1}\right)$ for rigid function symbols $f$ where $A_{1}, \ldots, A_{\# f+1}$ are either $D$ or $U$ each, depending on the sort of the respective argument of $f$ and the sort of the value of $f$.

$I(R) \in\left(A_{1} \times \ldots \times A_{\# R} \rightarrow\{0,1\}\right)$ for rigid relation symbols $R$ where $A_{1}, \ldots, A_{\# R}$ are chosen as for function symbols;

$I(c) \in(\tilde{\mathbf{I}}(T) \rightarrow A), I(f) \in\left(\tilde{\mathbf{I}}(T) \times A_{1} \times \ldots \times A_{\# f} \rightarrow A_{\# f+1}\right)$ and $I(R) \in\left(\tilde{\mathbf{I}}(T) \times A_{1} \times \ldots \times A_{\# R} \rightarrow\{0,1\}\right)$ for flexible $c, f$ and $R$ where the $A$ s are chosen as for rigid symbols;

$I(0)=0, I(+)=+$ and $I(=)$ is $=$ for $0,+$ and $=$ of either sort and its corresponding domain in $F . I(1)$ is the constant 1 from $U . I(\infty)=\infty$ and $I(\ell)=m$ like with $I T L$ interpretations.

Consider a non-empty set $\mathbf{W}$, a function $I$ on $\mathbf{W}$ into the set of the PITL interpretations of the fixed vocabulary $\mathbf{L}$ into the fixed frame $F$ and a function $P$ of type $\mathbf{W} \times T \times 2^{\mathbf{W}} \rightarrow U$. Let $I^{w}$ and $P^{w}$ abbreviate $I(w)$ and $\lambda \tau, X . P(w, \tau, X)$, respectively, for all $w \in \mathbf{W} . I^{w}$ and $P^{w}, w \in \mathbf{W}$, are intended to represent the set of behaviours and the associated probability distributions for every $\tau \in T$ in the F-based PITL models for $\mathbf{L}$ to be defined below.

Definition 2.3. Let $\tau \in T$. We define the equivalence relation $\equiv_{\tau}$ on $\mathbf{W}$ for all $\tau \in T$ by putting $w \equiv_{\tau} v$ iff

$I^{w}(s)=I^{v}(s)$ for all rigid symbols $s \in \mathbf{L}$, except possibly the individual variables; 
$I^{w}(s)\left(\sigma, d_{1}, \ldots, d_{\# s}\right)=I^{v}(s)\left(\sigma, d_{1}, \ldots, d_{\# s}\right)$ for all flexible $s \in \mathbf{L}$, all $d_{1}, \ldots, d_{\# s}$ from the appropriate domains and all $\sigma \in \tilde{\mathbf{I}}(T)$ such that $\max \sigma \leq \tau$;

$P^{w}\left(\tau^{\prime}, X\right)=P^{v}\left(\tau^{\prime}, X\right)$ for all $X \subseteq \mathbf{W}$ and all $\tau^{\prime} \leq \tau$.

Given $w \in \mathbf{W}$ and $\tau \in T$, we denote the set

$$
\left\{v \in \mathbf{W}: v \equiv_{\tau} w\right\}
$$

by $\mathbf{W}_{w, \tau}$.

Members of $\mathbf{W}$ which are $\tau$-equivalent stand for the same behaviour up to time $\tau$. If $\tau_{1}>\tau_{2}$, then $\equiv_{\tau_{1}} \subset \equiv_{\tau_{2}}$ and $w \equiv_{\infty} v$ holds iff $P^{w}=P^{v}$ and $I^{w}$ and $I^{v}$ agree on all symbols, except possibly some individual variables. $\mathbf{W}_{w, \tau}$ is the set of those $v \in \mathbf{W}$ which represent the probabilistic branching of $w$ from time $\tau$ onwards.

Definition 2.4. A general $P D C$ model for $\mathbf{L}$ is a tuple of the form $\langle F, \mathbf{W}, I, P\rangle$ where $F$, $\mathbf{W}, I$ and $P$ are as above and satisfy the following requirements for every $w \in \mathbf{W}$ :

$\mathbf{W}$ is closed under variants of interpretations. If $w \in \mathbf{W}, x$ is an individual variable from $\mathbf{L}$ and $a$ is in the domain from $F$ which corresponds to the sort of $x$, then there is a $v \in \mathbf{W}$ such that $P^{v}=P^{w}$ and $I^{v}=\left(I^{w}\right)_{x}^{a}$.

$P^{w}$ represents probability measures. The function $\lambda X . P^{w}(\tau, X)$ for every $w \in W$ and $\tau \in T$ is a finitely additive probability measure on the boolean algebra

$$
\left\langle 2^{\mathbf{W}}, \cap, \cup, \emptyset, \mathbf{W}\right\rangle \text {. }
$$

and satisfies the equality

$$
P^{w}(\tau, X)=P^{w}\left(\tau, X \cap \mathbf{W}_{w, \tau}\right) \text { for all } X \subseteq \mathbf{W},
$$

which means that $\lambda X . P^{w}(\tau, X)$ is required to be concentrated on the set $\mathbf{W}_{w, \tau}$.

Informally, a general PITL model is based on a set $\mathbf{W}$ of descriptions of infinite behaviours made by means of the ITL interpretations $I^{w}$ which are associated with each $w \in \mathbf{W}$. All the interpretations $I^{w}$ are into the same frame $F$ and are supposed to treat rigid symbols identically to express that, e. g., arithmetics is the same in all behaviours. It is assumed that, given a finite initial part of a behaviour $w$ until time $\tau$, the modelled system can proceed according to a description within the set $\mathbf{W}_{w, \tau}$ of the behaviours which are the same as $w$ up to time $\tau$. The probability for the system to choose a behaviour in $X \subseteq \mathbf{W}_{w, \tau}$ is $P^{w}(\tau, X)$.

Next we define term values $w_{\sigma}(t)$ and the satisfaction of formulas in PITL models. The definitions of term values, the modelling relation $\models$ and its associated notation $\llbracket . \rrbracket$ for terms, formulas, models and time intervals in PITL are given by the following clauses, where the components of the model $M$ are named as above:

Term values

$$
\begin{array}{lll}
w_{\sigma}(x) & =I^{w}(x) & \text { for variables } x \\
w_{\sigma}(c) & =I^{w}(c) & \text { for rigid } c \\
w_{\sigma}\left(f\left(t_{1}, \ldots, t_{\# f}\right)\right) & =I^{w}(f)\left(w_{\sigma}\left(t_{1}\right), \ldots, w_{\sigma}\left(t_{\# f}\right)\right) & \text { for rigid } f \\
w_{\sigma}(c) & =I^{w}(c)(\sigma) & \text { for flexible } c \\
w_{\sigma}\left(f\left(t_{1}, \ldots, t_{\# f}\right)\right) & =I^{w}(f)\left(\sigma, w_{\sigma}\left(t_{1}\right), \ldots, w_{\sigma}\left(t_{\# f}\right)\right) & \text { for flexible } f \\
w_{\sigma}(p(\psi)) & =P^{w}\left(\max \sigma, \llbracket \psi \rrbracket_{M, w, \sigma}\right) &
\end{array}
$$


Here $\llbracket \psi \rrbracket_{M, w, \sigma}$ stands for

$$
\left\{v \in \mathbf{W}_{w, \max \sigma}:\left(\forall v^{\prime} \in W\right)\left(P^{v^{\prime}}=P^{v} \wedge I^{v^{\prime}}=\left(I^{v}\right)_{x_{1}, \ldots, x_{n}}^{I^{w}\left(x_{1}\right), \ldots, I_{n}^{w}\left(x_{n}\right)} \rightarrow M, v^{\prime},[\min \sigma, \infty] \models \psi\right)\right\},
$$

where $x_{1}, \ldots, x_{n}$ are the free variables of $\psi$. This means that $\llbracket \psi \rrbracket_{M, w, \sigma}$ consists of the behaviours $v$ which are $\max \sigma$-equivalent to $w$ and satisfy $\psi$ at the infinite interval starting at $\min \sigma$.

Satisfaction of formulas

$$
\begin{array}{ll}
M, w, \sigma \not \models \perp & \\
M, w, \sigma \models R\left(t_{1}, \ldots, t_{\# R}\right) & \text { iff } I^{w}(R)\left(w_{\sigma}\left(t_{1}\right), \ldots, w_{\sigma}\left(t_{\# R}\right)\right)=1 \text { for rigid } R \\
M, w, \sigma \models R\left(t_{1}, \ldots, t_{\# R}\right) & \text { iff } I^{w}(R)\left(\sigma, w_{\sigma}\left(t_{1}\right), \ldots, w_{\sigma}\left(t_{\# R}\right)\right)=1 \text { for flexible } R \\
M, w, \sigma \models(\varphi \Rightarrow \psi) & \text { iff either } M, w, \sigma \not \models \varphi \text { or } M, w, \sigma \models \psi \\
M, w, \sigma \models(\varphi ; \psi) & \text { iff } M, w, \sigma_{1} \models \varphi \text { and } M, w, \sigma_{2} \models \psi \\
& \text { for some } \sigma_{1} \in \mathbf{I}^{\text {fin }}\left(T_{F}\right) \text { and } \sigma_{2} \in \tilde{\mathbf{I}}\left(T_{F}\right) \text { such that } \sigma_{1} ; \sigma_{2}=\sigma \\
M, w, \sigma \models \exists x \varphi & \text { iff } M, v, \sigma \models \varphi \text { for some } v \in \mathbf{W} \text { and some } a \text { from the }
\end{array}
$$$$
M, w, \sigma \models R\left(t_{1}, \ldots, t_{\# R}\right) \quad \text { iff } I^{w}(R)\left(\sigma, w_{\sigma}\left(t_{1}\right), \ldots, w_{\sigma}\left(t_{\# R}\right)\right)=1 \text { for flexible } R
$$$$
M, w, \sigma \models(\varphi ; \psi) \quad \text { iff } M, w, \sigma_{1} \models \varphi \text { and } M, w, \sigma_{2} \models \psi
$$$$
\text { for some } \sigma_{1} \in \mathbf{I}^{f i n}\left(T_{F}\right) \text { and } \sigma_{2} \in \tilde{\mathbf{I}}\left(T_{F}\right) \text { such that } \sigma_{1} ; \sigma_{2}=\sigma
$$$$
\text { domain of the sort of } x \text { such that } P^{v}=P^{w} \text { and } I^{v}=\left(I^{w}\right)_{x}^{a}
$$

Obviously $M, w, \sigma \models \psi$ iff $\left\langle F, I^{w}\right\rangle$, $[\min \sigma, \infty] \models_{I T L} \psi$ as in non-probabilistic ITL for $\psi$ with no occurrence of probability terms.

The probability functions $\lambda X . P^{w}(\tau, X)$ for $w \in \mathbf{W}$ and $\tau \in T$ in general PITL models $M=\langle F, \mathbf{W}, I, P\rangle$ are needed just as much as they provide values for probability terms. That is why these functions need not be defined on the entire algebra (2.1). Indeed, it is sufficient for $\lambda X . P^{w}(\tau, X)$ to be defined on the (generally smaller) algebra

$$
\left\langle\left\{\llbracket \psi \rrbracket_{M, w, \sigma}: \psi \in \mathbf{L}, \sigma \in \tilde{\mathbf{I}}(T), \max \sigma=\tau\right\}, \cap, \cup, \emptyset, \mathbf{W}_{w, \tau}\right\rangle,
$$

which we denote by $\mathbf{B}_{M, w, \tau}$. This observation justifies the broadening of the definition of general PITL models as follows.

Amendment to Definition 2.4 Structures of the form $M=\langle F, \mathbf{W}, P, I\rangle$ from Definition 2.4. but with their probability functions $\lambda X . P^{w}(\tau, X)$ defined just on the respective algebras $\mathbf{B}_{M, w, \tau}$, are general PITL models too.

Example A PITL model $M_{\mathbf{A}}=\left\langle F_{\mathbf{R}}, \mathbf{W}, P, I\right\rangle$ which is based on the real-time frame $F_{\mathbf{R}}$ and describes the working of a given probabilistic automaton $\mathbf{A}$ of the form (1.2) from Definition 1.3 can be defined as follows. The vocabulary of $M_{\mathbf{A}}$ includes of the mandatory symbols $0,+, \ell, \ldots$, the transitions $a \in A$ as flexible 0 -ary predicate letters, and the choice probabilities $q_{a}$ as rigid constants. As for the duration probability densities $p_{a}$, it is convenient to have rigid unary function symbols $P_{a}$ which denote the functions $\lambda \tau \cdot \int_{0}^{\tau} p_{b}(t) d t$. The vocabulary does not provide direct reference to the states of $\mathbf{A}$ as done in $P D C$; behaviour is instead described in terms of transitions whose beginnings and ends mark the times of state change. Every possible behaviour (1.3) is described by a $w \in \mathbf{W}$ such that $I^{w}\left(a_{i}\right)\left(\left[\sum_{j<i} d_{j}, \sum_{j \leq i} d_{j}\right]\right)=1 . I^{w}(a)\left(\left[\tau_{1}, \tau_{2}\right]\right)=1$ holds only if $\left[\tau_{1}, \tau_{2}\right]$ is one of the intervals 
$\left[\sum_{j<i} d_{j}, \sum_{j \leq i} d_{j}\right], i<\omega$, and $a$ is the corresponding $a_{i}$. Given $w \in \mathbf{W}$ and $\tau \in \mathbf{R}_{+}, P^{w}(\tau, X)$ is defined as the probability for the finite behaviour described by $w$ up to time $\tau$ to develop into an infinite behaviour from $X$. For instance, let

$$
\left\langle F_{\mathbf{R}}, I^{w}\right\rangle,[0, \tau] \models(\top ; a),
$$

which means that the interval $[0, \tau]$ accommodates a finite sequence of transitions which ends at $a$ and a new transition is to begin at time $\tau$. Then, if $b \in A$ and $b^{-}=a^{+}, P^{w}$ satisfies the equality

$$
P^{w}\left(\tau, \llbracket(b \wedge x \leq \ell \wedge \ell \leq y ; \top) \rrbracket M_{\mathbf{A}, w,[\tau, \tau]}\right)=q_{b} \int_{I^{w}(x)}^{I^{w}(y)} p_{b}(t) d t .
$$

Here $\llbracket(b \wedge x \leq \ell \wedge \ell \leq y ; \top) \rrbracket_{M_{\mathbf{A}}, w,[\tau, \tau]}$ is the set of all the behaviours in which the part of $w$ until time $\tau$ is continued by transition $b$ and the duration of $b$ is in the range $\left[I^{w}(x), I^{w}(y)\right]$. The equality (2.3) describes the probability for such a development to take place. If the source state of $b$ is $s_{0}$, then (2.3) holds for $\tau=0$ and all $w$ as well. (2.3) entails that the formula

$$
\neg\left(\top ; a ; \ell=0 \wedge p((b \wedge x \leq \ell \wedge \ell \leq y ; \top)) \neq q_{b} .\left(P_{b}(y)-P_{b}(x)\right)\right),
$$

is valid in $M_{\mathbf{A}}$. This formula means that the probability for a behaviour satisfying $(b \wedge$ $x \leq \ell \wedge \ell \leq y ; \top)$ to take place after $(\top ; a)$ is $q_{b} \cdot\left(P_{b}(y)-P_{b}(x)\right)$, which, by the chosen interpretation of $P_{b}$, is equal to the righthand side of (2.3).

Describing probabilistic real-time automata in a system of infinite interval probabilistic duration calculus which corresponds to PITL is the topic of Section 6.3.

We conclude the definition of PITL semantics with a remark on the underlying model of time. As mentioned in the introduction, PDC and PITL are essentially branching-time interval logics. An alternative way to introduce the semantics of PITL could be to use partially ordered time domains $\langle T, \leq\rangle$ with some additional conditions on their maximal linearly ordered subsets. Given a PITL model $\langle F, \mathbf{W}, I, P\rangle$ as described above, we can construct the corresponding partially ordered time domain by taking

$$
\left\{\left\langle\tau, \mathbf{W}_{w, \tau}\right\rangle: \tau \in T, w \in \mathbf{W}\right\}
$$

as the set of time points and defining the partial ordering by the clause

$$
\left\langle\tau_{1}, W_{1}\right\rangle \leq\left\langle\tau_{2}, W_{2}\right\rangle \text { iff } \tau_{1} \leq \tau_{2} \text { and } W_{1} \supseteq W_{2} .
$$

The chosen way to define PITL models saves us the need to reformulate results on ITL which are essentially linear-time and are therefore known in the literature just for the sake of notation differences.

\section{A PRoOF SYSTEM FOR PITL}

In this section we propose axioms and a proof rule for PITL. If added to the complete proof system for ITL with infinite intervals from [WX04] given in Section 1.1.4, these axioms and the rule form a system which is complete for PITL with respect to its abstract semantics introduced in Section 2.2. This is demonstrated in Section 4. Most of our axioms and rule are modifications of those for $P N L$ from [Gue00]. The modifications were made

to account for the use of infinite intervals instead of the $N L$ expanding modalities. Some simple infinite-interval-specific properties of $p($.$) are handled by completely new axioms.$ 


\subsection{The system.}

\section{Extensionality}

$$
\begin{aligned}
& \left(P_{;}\right) \quad(\ell=x ; p(\psi)=y) \Rightarrow p((\ell=x ; \psi))=y \\
& \left(P_{\infty}\right) \quad \ell=\infty \Rightarrow(\varphi \Leftrightarrow p(\varphi)=1) \\
& \left(P_{\leq}\right) \quad \frac{\vdash(\varphi ; \ell=\infty) \Rightarrow(\psi \Rightarrow \chi)}{\vdash \varphi \wedge \ell<\infty \Rightarrow p(\psi) \leq p(\chi)}
\end{aligned}
$$

Arithmetics of probabilities

$$
\begin{array}{ll}
\left(P_{\perp}\right) & p(\perp)=0 \\
\left(P_{\top}\right) & p(\top)=1 \\
\left(P_{+}\right) & p(\varphi)+p(\psi)=p(\varphi \vee \psi)+p(\varphi \wedge \psi)
\end{array}
$$

$P$; expresses that the probability function $P_{\langle I, P\rangle, \max \sigma}$ which is used to evaluate $I_{\sigma}(p(\psi))$ depends on the end point $\max \sigma$ and not on the whole reference interval $\sigma$. $P_{\infty}$ means that having the entire future as the reference interval renders all properties deterministic: no alternative behaviours are possible "from $\infty$ on"; the interpretations $I^{\prime}$ from $\left\langle I^{\prime}, P^{\prime}\right\rangle \in$ $\mathbf{W}_{\langle I, P\rangle, \infty}$ can differ from $I$ only on individual variables and such differences are disregarded in the definition (2.2) of $\llbracket \varphi \rrbracket_{M,\langle I, P\rangle, \sigma}$ for all intervals $\sigma$. The rule $P_{\leq}$means that if a property $\chi$ is a logical consequence of another property $\psi$, then the probability of $\chi$ is at least as big as that of $\psi$. The probabilities of $\psi$ and $\chi$ are compared in the context of a finite-interval condition $\varphi$. The case of an infinite-interval condition $\varphi$ is handled by axiom $P_{\infty}$. The axioms $P_{\perp}, P_{\top}$ and $P_{+}$are self-explanatory. The correctness of the axioms and the rule is straightforward. The use of $\vdash$ in $P_{\leq}$is to emphasize that we intend to apply this rule only to theorems. The maximal consistent sets of formulas which take part in our completeness argument for this proof system below need not be closed under $P_{\leq}$.

The rule $P_{\leq}$can be classified under the category of probability arithmetics as well, because of the meaning of $\leq$, which is defined by (1.1). However, we find its role as an extensionality rule, which is further highlighted by the derived rule PITL1 below, to be more important.

3.2. Some useful PITL theorems and a derived rule. The PITL theorems PITL2 and PITL3 and the derived rule PITL1 below are used in proofs in the rest of the paper. PITL4 is included to highlight the role of infinite intervals in the semantics of probability terms and the effect of $\tau$-equivalence on probabilities, respectively.

$$
\begin{array}{ll}
\left(P_{\leq}^{\infty}\right) & \frac{(\varphi ; \ell=\infty) \vee(\varphi \wedge \ell=\infty) \Rightarrow(\psi \Rightarrow \chi)}{\varphi \Rightarrow p(\psi) \leq p(\chi)} \\
(\text { PITL1) } & \frac{\varphi \Leftrightarrow \psi}{p(\varphi)=p(\psi)} \\
(\text { PITL2) } & p(\varphi)+p(\neg \varphi)=1 \\
(\text { PITL3) } & p(\varphi)<p(\psi) \Rightarrow p(\psi \wedge \neg \varphi) \neq 0 \\
(\text { PITL4) } & p(\varphi)=p(\varphi \wedge \ell=\infty)
\end{array}
$$


Here follows a derivation for $P_{\leq}^{\infty}$. The purely $I T L$ parts are skipped and marked "ITL" for the sake of brevity. Applications of the axioms $U 1-U 7$ for arithmetics on probability domains are skipped without comments.

$$
\begin{aligned}
& 1 \quad(\varphi ; \ell=\infty) \Rightarrow(\psi \Rightarrow \chi) \\
& 2 \varphi \wedge \ell<\infty \Rightarrow p(\psi) \leq p(\chi) \\
& 3 \ell=\infty \wedge \varphi \Rightarrow(p(\psi)=0 \wedge p(\chi)=0) \\
& \vee(p(\psi)=0 \wedge p(\chi)=1) \\
& \vee(p(\psi)=1 \wedge p(\chi)=1) \\
& 4 \varphi \wedge \ell=\infty \Rightarrow p(\psi) \leq p(\chi) \\
& \text { assumption, ITL } \\
& 1, P_{\leq} \\
& \text {assumption, } P_{\infty}, \text { PITL2 } \\
& 5 \quad \ell<\infty \vee \ell=\infty \\
& \text { 3, ITL } \\
& 6 \varphi \Rightarrow p(\psi) \leq p(\chi) \\
& 2,4,5
\end{aligned}
$$

PITL4 is obtained by applying $P_{\leq}^{\infty}$ to the ITL theorems

$$
\begin{aligned}
& (\top ; \ell=\infty) \vee(\top \wedge \ell=\infty) \Rightarrow(\varphi \Rightarrow \varphi \wedge \ell=\infty) \text { and } \\
& (\top ; \ell=\infty) \vee(\top \wedge \ell=\infty) \Rightarrow(\ell=\infty \wedge \varphi \Rightarrow \varphi) .
\end{aligned}
$$

The rule PITL1 is proved by two applications of $P_{\leq}^{\infty}$ too. The proofs for PITL2 and PITL3 below are included as simple examples of the working of the axioms about arithmetics of probabilities.

\section{PITL2:}

$\begin{array}{lll}1 & \varphi \wedge \neg \varphi \Leftrightarrow \perp & I T L \\ 2 & p(\varphi \wedge \neg \varphi)=p(\perp) & 1, \text { PITL1 } \\ 3 & p(\varphi \wedge \neg \varphi)=0 & 2, P_{\perp} \\ 4 & \varphi \vee \neg \varphi \Leftrightarrow \top & I T L \\ 5 & p(\varphi \vee \neg \varphi)=p(\top) & 4, P I T L 1 \\ 6 & p(\varphi \wedge \neg \varphi)=1 & 5, P_{\top} \\ 7 & p(\varphi)+p(\neg \varphi)=p(\varphi \wedge \neg \varphi)+p(\varphi \wedge \neg \varphi) & P_{+} \\ 8 & p(\varphi)+p(\neg \varphi)=1 & 2,6,7, I T L\end{array}$

PITL3:

$\begin{array}{lll}1 & p(\psi) \leq p(\varphi \vee \psi) & P_{\leq}^{\infty} \\ 2 & p(\varphi)+p(\psi \wedge \neg \varphi)=p(\varphi \wedge \psi \wedge \neg \varphi)+p(\varphi \vee \psi \wedge \neg \varphi) & P_{+} \\ 3 & p(\varphi)+p(\psi \wedge \neg \varphi)=p(\varphi \vee \psi) & 2, \text { PITL1, } P_{\perp} \\ 4 & p(\varphi)<p(\psi) \Rightarrow p(\varphi)<p(\varphi \vee \psi) & 1 \\ 5 & p(\varphi)<p(\psi) \Rightarrow p(\psi \wedge \neg \varphi) \neq 0 & 3,4\end{array}$

\section{Completeness of the PRoOf System for PITL}

In this section we show that the proof system for PITL from Section 3 is complete. To exploit the full potential of the abstract semantics of PITL, we prove a strong completeness theorem. It states that every consistent set of PITL formulas has a model. This is convenient for the study of further extensions of the logic whose syntactic elements can be represented by adding infinitely many non-logical symbols and axioms about them, or when a modelled system is described using infinitely many formulas. 
The main step in this proof is the construction of what is known in model theory as the elementary diagram $\Delta$ of a PITL model $M$ for an arbitrary given set of PITL formulas $\Gamma$ which is consistent in the proposed proof system for PITL. $\Delta$ is a description of $M$ in a PITL language whose vocabulary has names for all the elements of $M$. To avoid repeating the technical steps which are not specific to the probability operator of PITL and can be found in the completeness proof for (non-probabilistic) ITL with infinite intervals from WX04, we introduce a translation of the involved PITL languages into corresponding ITL languages with appropriate vocabularies and use it to view subsets of the constructed diagram and the whole diagram as complete Henkin theories in (non-probabilistic) ITL as well.

The model $M$ that we construct is very similar to a canonical model. We stop short of calling it canonical, because of the dedicated technique which is used to build the behaviour representations $v$ which are needed to populate the sets $\llbracket \varphi \rrbracket_{M, w, \sigma}$ for $\varphi, \sigma$ and $w$ such that $M, w, \sigma \models p(\varphi) \neq 0$ is supposed to hold.

Without losing generality, we consider only sets of formulas $\Gamma$ which contain $\ell=\infty$. This way we restrict ourselves to seeking the satisfaction of $\Gamma$ at an infinite interval. The satisfaction of a consistent $\Gamma$ which is not consistent with $\ell=\infty$ can be achieved through the satisfaction of

$$
\{\ell=\infty\} \cup\{(\gamma \wedge \ell=c ; \top): \gamma \in \Gamma\}
$$

where $c$ is some fresh rigid constant.

The completeness argument involves the application of some non-trivial results about interpolation in $I T L$. We present them first.

4.1. Interval-related and Craig interpolation in ITL with infinite intervals. Interval-related interpolation for $I T L$ with finite intervals, $N L$ and a subset of $D C$ with finite intervals and projection onto state were formulated and proved in Gue01, Gue04b]. Craig interpolation was shown to hold for these logics there too. Here we just formulate intervalrelated interpolation for $I T L$ with infinite intervals in the special form which is convenient for our completeness argument.

Let $\mathbf{L}$ and $\mathbf{L}^{\prime}$ be two vocabularies for $I T L$ with infinite intervals. Let $\mathbf{L}$ and $\mathbf{L}^{\prime}$ share their rigid symbols, including the individual variables, and let the only flexible symbol occurring in both $\mathbf{L}$ and $\mathbf{L}^{\prime}$ be $\ell$. Let there be a bijection between the flexible symbols from $\mathbf{L} \backslash\{\ell\}$ and those from $\mathbf{L}^{\prime}$ such that the symbol $s^{\prime}$ from $\mathbf{L}^{\prime}$ which corresponds to $s \in \mathbf{L}$ is of the same kind and arity as $s$. Let $\varphi^{\prime}$ denote the result of replacing each flexible symbol $s \in \mathbf{L} \backslash\{\ell\}$ in a formula $\varphi$ written in $\mathbf{L}$ by the corresponding $s^{\prime} \in \mathbf{L}^{\prime}$.

Theorem 4.1. Let $\Phi$ be a finite set of formulas and $\varphi$ and $\psi$ be two more formulas, all written in $\mathbf{L}$. Let $c$ be a rigid constant in $\mathbf{L}$. Let

$$
\left(\ell=c \wedge \square \forall \bigwedge_{\chi \in \Phi}\left(\chi \Leftrightarrow \chi^{\prime}\right) ; \ell=\infty\right) \Rightarrow\left(\varphi \Rightarrow \psi^{\prime}\right)
$$

be theorem of ITL with infinite intervals. Then there is a formula $\theta$ written in $\mathbf{L}$ such that

$$
\varphi \wedge c<\infty \wedge \ell=\infty \Rightarrow(\ell=c \wedge \theta ; \ell=\infty) \text { and }\left(\ell=c \wedge \theta^{\prime} ; \ell=\infty\right) \Rightarrow \psi^{\prime}
$$

are theorems of ITL as well.

We use the standard form of Craig interpolation: 
Theorem 4.2. Let $\mathbf{L}_{1}$ and $\mathbf{L}_{2}$ be two ITL vocabularies. Let $\varphi_{i}$ be a formula of ITL with infinite intervals written in the vocabulary $\mathbf{L}_{i}, i=1,2$, and

$$
\varphi_{1} \Rightarrow \varphi_{2}
$$

be a theorem of ITL with infinite intervals. Then there is a formula $\theta$ written in the vocabulary $\mathbf{L}_{1} \cap \mathbf{L}_{2}$ such that both

$$
\varphi_{1} \Rightarrow \theta \text { and } \theta \Rightarrow \varphi_{2}
$$

are such theorems.

The proofs of the two interpolation theorems are simple variants of those of the theorems known from [Gue01, which in their turn follow the pattern of the model-theoretic proof of Craig interpolation that can be seen in, e.g., CK73.

\subsection{Consistency in PITL.}

Definition 4.3. Given an $I T L(P I T L)$ vocabulary $\mathbf{L}, I T L_{\mathbf{L}}\left(P I T L_{\mathbf{L}}\right)$ denotes the set of the theorems of $I T L(P I T L)$ written in a given vocabulary $\mathbf{L}$. Given $\mathbf{L}$ and a set of formulas $\Gamma$ written in $\mathbf{L}, C n_{\mathbf{L}, I T L}(\Gamma)\left(C n_{\mathbf{L}, P I T L}(\Gamma)\right)$ denotes the set of formulas written in $\mathbf{L}$ which can be proved using formulas from $I T L_{\mathbf{L}} \cup \Gamma\left(P I T L_{\mathbf{L}} \cup \Gamma\right)$ and the propositional logic rule Modus Ponens $\varphi, \varphi \Rightarrow \psi / \psi$.

Definition 4.4. A set of $I T L(P I T L)$ formulas $\Gamma$ written in a vocabulary $\mathbf{L}$ is consistent if $\perp \notin C n_{\mathbf{L}, I T L}(\Gamma)\left(\perp \notin C n_{\mathbf{L}, P I T L}(\Gamma)\right)$. A consistent $\Gamma$ is maximal in $\mathbf{L}$ if it has no consistent proper supersets of formulas written in $\mathbf{L}$.

Just like in first-order predicate logic, a set of formulas $\Gamma$ has witnesses in some set of rigid constants $C$ if for every existential formula $\exists x \varphi \in \Gamma$ there is a witness $c \in C$ such that $[c / x] \varphi \in \Gamma$.

Here follows the Lindenbaum Lemma for PITL as known from numerous predicate and modal logics:

Theorem 4.5. Let $\Gamma$ be a consistent set of formulas PITL written in some vocabulary $\mathbf{L}$ and $C$ be a countably-infinite set which consists of infinitely many fresh constants of both the sort of durations and the sort of probabilities. Then there is a maximal consistent set of formulas written in $\mathbf{L} \cup C$ which contains $\Gamma$ and has witnesses in $C$.

We omit the proof for PITL, because it is the same as that for ITL with abstract semantics and finite intervals which can be seen in Dut95a]. The proof for ITL with infinite intervals was omitted in WX04 for the same reason.

4.3. A vocabulary for the elementary diagram $\Delta$ for the PITL model $M$. The PITL vocabulary $\mathbf{L}_{D}$ which we introduce next is structured so that a PITL model $M$ for the extension of some given PITL vocabulary $\mathbf{L}$ by a countable set of fresh rigid constants that we construct below can be fully described in it in terms of rather simple quantifierand variable-free formulas which can be regarded as making up a diagram $\Delta$ for $M$ in the model-theoretic sense. $\mathbf{L}_{D}$ contains rigid constants to name all the elements of the duration domain and the probability domain of $M$ and a separate set of flexible symbols to describe the behaviour of the flexible symbols of $\mathbf{L}$ in each interpretation from $M$. Indeed, we construct an elementary diagram for $M$ in $\mathbf{L}_{D}$, which consists of all the formulas in $\mathbf{L}_{D}$ which hold at some infinite interval in $M$ under the convention that formulas written in the 
various sets of flexible symbols mentioned above are understood to hold at the respective interpretations.

$\mathbf{L}_{D}$ is the union of the following sets of symbols:

1. The rigid symbols of $\mathbf{L}$, including the individual variables, and the mandatory flexible constant $\ell$.

2. Two countably-infinite sets of fresh rigid constants $C^{d}$ and $C^{p}$ of the sorts of durations and probabilities, respectively, whose structure is explained below.

3. The fresh flexible symbols $s^{\nu}, \nu \in S$, of the same kind and arity as $s$, for each flexible $s \in \mathbf{L} \backslash\{\ell\}$. The countably-infinite index set $S$ is defined below.

$C^{d}$ and $C^{p}$ are assumed to be the countably-infinite disjoint unions of some countably infinite sets $C_{k}^{d}$ and $C_{k}^{p}, k<\omega$, respectively. Similarly, $S$ is assumed to be the countablyinfinite union of the sets $S_{k}, k<\omega$. We denote $\bigcup_{i \leq k} C_{i}^{d}, \bigcup_{i<k} C_{i}^{p}$ and $\bigcup_{i \leq k} S_{i}$ by $C_{\leq k}^{d}, C_{\leq k}^{p}$ and $S_{\leq k}$, respectively, for all $k<\omega$. We denote the vocabulary which consists of the rigid symbols of $\mathbf{L}, \ell$, the rigid constants from $C_{\leq k}^{d}$ and $C_{\leq k}^{p}$ and the flexible symbols $s^{\nu}$ for $\nu \in S_{\leq k}$ by $\mathbf{L}_{\leq k}$ for all $k<\omega$. We denote the extension of $\mathbf{L}_{\leq k}$ by the flexible symbols $s^{\nu}$ for $\nu \in S_{\leq k+1}$ by $\mathbf{L}_{\leq k+1}^{\prime}$.

The set $S_{0}$ is the singleton $\{\langle\rangle\}$, which consists of the empty list \langle\rangle .

$$
S_{k+1}=\left\{\langle\nu, c, \varphi\rangle: \nu \in S_{\leq k}, c \in C_{\leq k}^{d}, \varphi \text { is written in } \mathbf{L}_{\leq k}\right\} \text { for all } k<\omega .
$$

In the construction of $\Delta$ below, given a $\nu \in S, A^{\nu}$ stands for the result of replacing the flexible symbols $s \in \mathbf{L} \backslash\{\ell\}$ in a term or formula $A$ written in the vocabulary $\mathbf{L} \cup C^{d} \cup C^{p}$ by their corresponding symbols $s^{\nu}$. We denote the vocabulary which consists of the rigid symbols of $\mathbf{L}$, including the individual variables, $\ell$ and the flexible symbols $s^{\nu}$ for some fixed $\nu \in S$ and all flexible $s \in \mathbf{L} \backslash\{\ell\}$ by $\mathbf{L}^{\nu}$.

4.4. A translation of PITL formulas into $I T L$. Let $\mathbf{L}$ be a PITL vocabulary. We define its corresponding vocabulary $\mathbf{L}_{I T L}$ for two-sorted (non-probabilistic) ITL with infinite intervals with the sorts of durations and probabilities as in PITL. Roughly speaking, $\mathbf{L}_{I T L}$ is an extension of $\mathbf{L}$ by flexible constants and function symbols which are meant to simulate probability terms. Here follows the precise definition.

Definition 4.6. $\mathbf{L}_{I T L}$ is the union of the vocabularies $\mathbf{L}_{I T L, k}, k<\omega . \mathbf{L}_{I T L, 0}$ is $\mathbf{L}$. Given $\mathbf{L}_{I T L, i}, i \leq k, \mathbf{L}_{I T L, k+1}$ is the set of flexible constants and function symbols

$$
\left\{\mathrm{p}_{\varphi}: \varphi \text { is a formula written in } \bigcup_{i \leq k} \mathbf{L}_{I T L, k} \text { and contains at least one symbol from } \mathbf{L}_{I T L, k}\right\} \text {. }
$$

The values of the symbols $\mathrm{p}_{\varphi}$ are of the probability sort. If $\varphi$ has no free variables, then $\mathrm{p}_{\varphi}$ is a flexible constant. Otherwise $\mathrm{p}_{\varphi}$ is a flexible function symbol whose arity is $|F V(\varphi)|$ and the sort of the $i$ th argument of $\mathrm{p}_{\varphi}$ is that of the $i$ th free variable of $\varphi$ with respect to some fixed ordering of these variables, $i=1, \ldots,|F V(\varphi)|$.

Next we define a translation $\mathrm{t}$ of PITL terms and formulas written in $\mathbf{L}$ into ITL formulas written in $\mathbf{L}_{I T L}$. The goal of $\mathrm{t}$ is to systematically replace the occurrences of probability terms by terms built using the corresponding constant and function symbols from Definition 4.6. To achieve this, $\mathrm{t}$ works by the following rule:

$$
\left[p\left(\psi_{1}\right) / z_{1}, \ldots, p\left(\psi_{n}\right) / z_{n}\right] A
$$


where denotes $A$ a term or formula with no probability terms is translated into

$$
\left[\mathrm{p}_{\mathrm{t}\left(\psi_{1}\right)}\left(x_{1,1}, \ldots, x_{1, m_{1}}\right) / z_{1}, \ldots, \mathrm{p}_{\mathrm{t}\left(\psi_{1}\right)}\left(x_{n, 1}, \ldots, x_{n, m_{n}}\right) / z_{n}\right] A
$$

where $x_{i, 1}, \ldots, x_{i, m_{i}}$ are the free variables of $\psi_{i}$ in the fixed ordering mentioned above, $i=1, \ldots, n$. If $F V(\psi)=\emptyset$, then the expression $\mathrm{p}_{\mathrm{t}\left(\psi_{i}\right)}\left(x_{i, 1}, \ldots, x_{i, m_{i}}\right)$ denotes just the flexible constant $\mathrm{p}_{\mathrm{t}\left(\psi_{i}\right)}$.

Example If there are no probability terms in $\varphi$ and $F V(\varphi)=x_{1}$, then $\mathrm{t}(p(\varphi))$ is the term $\mathrm{p}_{\varphi}\left(x_{1}\right)$ and $\mathrm{t}\left(p\left(\left(\ell=x_{2} ; p(\varphi)<p(\neg \varphi)\right)\right)\right)$ is $\mathrm{p}_{\left(\ell=x_{2} ; \mathrm{p}_{\varphi}\left(x_{1}\right)<\mathrm{p}_{\neg \varphi}\left(x_{1}\right)\right)}\left(x_{1}, x_{2}\right)$.

Every term and formula can be represented in the form (4.2) in a unique way up to renaming the distinct variables $z_{1}, \ldots, z_{n}$, if we assume that all of these variables have free occurrences in $A$ and that the formulas $\psi_{1}, \ldots, \psi_{n}$ are all different. The semantical correctness of the substitution in (4.2) and (4.3) is not relevant to this definition of $t$. Given a set of PITL formulas $\Gamma$, we denote $\{\mathrm{t}(\gamma): \gamma \in \Gamma\}$ by $\mathrm{t}(\Gamma)$.

Terms built using the function symbols $\mathrm{p}_{\psi}$ from $\mathbf{L}_{I T L}$ in translations of PITL formulas always have the free variables of $\psi$ as their argument terms. That is why formulas written in $\mathbf{L}_{I T L}$ which contain $\mathrm{p}_{\psi}$ in terms of other forms are not in the range of $\mathrm{t}$. However, they always have equivalents of the form $\mathrm{t}(\varphi)$ for appropriate PITL formulas $\varphi$ written in $\mathbf{L}$. To realise that, note that if $F V(\psi)=\left\{x_{1}, \ldots, x_{n}\right\}$ and $y_{1}, \ldots, y_{n}$ are $n$ fresh variables of the appropriate sorts, then $\mathrm{p}_{\psi}\left(t_{1}, \ldots, t_{n}\right)=z$ is equivalent to

$$
\exists y_{1} \ldots \exists y_{n}\left(\bigwedge_{i=1}^{n} t_{i}=y_{i} \wedge \exists x_{1} \ldots \exists x_{n}\left(\bigwedge_{i=1}^{n} y_{i}=x_{i} \wedge \mathrm{p}_{\psi}\left(x_{1}, \ldots, x_{n}\right)=z\right)\right) .
$$

Furthermore, every formula written in $\mathbf{L}_{I T L}$ has an equivalent in which the terms of the form $\mathrm{p}_{\psi}\left(t_{1}, \ldots, t_{n}\right)$ appear only in atomic formulas of the form $\mathrm{p}_{\psi}\left(t_{1}, \ldots, t_{n}\right)=z$ where $z$ can be chosen to be different from $x_{1}, \ldots, x_{n}$.

Now we turn to the correspondence between derivability in PITL and ITL with infinite intervals.

Proposition 4.7. Let $\mathbf{L}$ be a PITL vocabulary and $\Gamma$ be a set of formulas written in $\mathbf{L}$. Then

$$
\mathrm{t}\left(C n_{\mathbf{L}, P I T L}(\Gamma)\right)=C n_{\mathbf{L}_{I T L}, I T L}\left(\mathrm{t}\left(P I T L_{\mathbf{L}} \cup \Gamma\right)\right) .
$$

Proof. Simple induction on the construction of proofs.

Corollary 4.8. A set of PITL formulas $\Gamma$ written in a vocabulary $\mathbf{L}$ is consistent iff $C n_{\mathbf{L}_{\text {ITL }}, I T L}\left(P I T L_{\mathbf{L}} \cup \Gamma\right)$ is consistent.

Proof. $\mathrm{t}(\perp)$ is $\perp$.

4.5. The weakened proof system $P I T L^{-}$. The model $M$ constructed below is for $\mathbf{L} \cup$ $C^{d} \cup C^{p}$. It contains one class of $w \in \mathbf{W}$ which are the same except possibly for the interpretations $I^{w}$ of some individual variables for every $\nu \in S$. Let $w_{\nu}$ denote a representative for the class of interpretations corresponding to $\nu$. Then $I^{w_{\nu}}(s)$ is defined by the formulas from the diagram $\Delta$ for $M$ which describe $s^{\nu}$ for all flexible $s \in \mathbf{L} \backslash\{\ell\}$. We are interested in having a set of formulas $\Gamma$ which contains the formula $\ell=\infty$ satisfied at some infinite interval $\left[\tau_{0}, \infty\right]$ and some interpretation $I$ in $M$. Our construction of $M$ provides that if 
$c \in C^{d}$ and $\tau_{1}$ is defined by the equality $m\left(\left[\tau_{0}, \tau_{1}\right]\right)=I^{w_{\nu}}(c)$ in $M$, then $w_{\nu}$ and $w_{\langle\nu, c, \varphi\rangle}$ are related as follows:

$$
\begin{aligned}
& \text { If } M, w_{\nu},\left[\tau_{0}, \tau_{1}\right] \models p(\varphi) \neq 0 \text { and } F V(\varphi)=\left\{x_{1}, \ldots, x_{n}\right\}, \text { then } w_{\nu} \equiv_{\tau_{1}} w_{\langle\nu, c, \varphi\rangle} \\
& \text { and } M, v,\left[\tau_{0}, \infty\right] \models \varphi \text { for some } v \text { such that } I^{v}=\left(I^{w_{\langle\nu, c, \varphi\rangle}}\right)_{x_{1}}^{I_{\nu}\left(x_{1}\right), \ldots, I_{\nu}\left(x_{n}\right)} \text { and } \\
& P^{v}=P^{w_{\langle\nu, c, \varphi\rangle}} .
\end{aligned}
$$

This means that $w_{\langle\nu, c, \varphi\rangle} \in \llbracket \varphi \rrbracket_{M, w_{\nu},\left[\tau_{0}, \tau_{1}\right]}$.

Furthermore, we are interested in enforcing PITL local logical consequence at each particular $w \in \mathbf{W}$, but not across different $w$. That is why in the construction of $\Delta$ below we restrict the applicability of the $P I T L$-specific axioms $P_{;}, P_{\infty}, P_{\perp}, P_{\top}$ and $P_{+}$and rule $P_{\leq}$from Section 3 in sets of formulas written in $\mathbf{L}_{D}$. We allow only instances of $P_{;}, P_{\infty}$, $P_{\leq}, P_{\perp}, P_{\top}$ and $P_{+}$in which all flexible symbols except $\ell$ have the same superscript $\nu \in S$. The resulting weakened proof system is tied to the vocabulary $\mathbf{L}_{D}$. We denote it and the set of its theorems written in a given sub-vocabulary $\mathbf{L}^{\prime}$ of $\mathbf{L}_{D}$ by $P I T L^{-}$and $P I T L_{\mathbf{L}^{\prime}}^{-}$, respectively. Theorem 4.5 applies to consistency with $P I T L_{\mathbf{L}^{\prime}}^{-}$without change. Similarly, we have the following variant of Proposition 4.7,

Proposition 4.9. Let $\mathbf{L}^{\prime}$ be a sub-vocabulary of $\mathbf{L}_{D}$ and $\Gamma$ be a set of formulas written in $\mathbf{L}^{\prime}$. Then

$$
\mathrm{t}\left(C n_{\mathbf{L}^{\prime}, P I T L^{-}}(\Gamma)\right)=C n_{\mathbf{L}_{I T L}^{\prime}, I T L}\left(\mathrm{t}\left(P I T L_{\mathbf{L}^{\prime}}^{-} \cup \Gamma\right)\right) .
$$

We also use the following somewhat more involved technical consequence of the restricted use of the instances of $P_{;}, P_{\infty}, P_{\leq}, P_{\perp}, P_{\top}$ and $P_{+}$and the restricted application of $P_{\leq}$.

Lemma 4.10. Let $\alpha \in P I T L_{\mathbf{L}^{\prime}}^{-}$for some sub-vocabulary $\mathbf{L}^{\prime}$ of $\mathbf{L}_{D}$. Let $C$ be the set of the rigid constants of $\mathbf{L}^{\prime}$. Then there exist finitely many superscripts $\nu_{1}, \ldots, \nu_{n} \in S$ and theorems $\beta_{i} \in P I T L_{\mathbf{L}^{\nu} \cup C}, i=1, \ldots, n$, such that the formula

$$
\bigwedge_{i=1}^{n} \square \forall \beta_{i} \Rightarrow \alpha
$$

is provable without the use of $P_{;}, P_{\infty}, P_{\perp}, P_{\top}$ and $P_{+}$and $P_{\leq}$, that is, essentially in (non-probabilistic) ITL with infinite intervals.

Proof. Consider a PITL $L^{-}$proof of $\alpha$ in $\mathbf{L}^{\prime}$. Let $\nu_{1}, \ldots, \nu_{n}$ be all the superscripts of flexible symbols occurring in formulas from this proof. If a formula $\beta$ from the proof is written in the vocabulary $\mathbf{L}^{\nu_{i}} \cup C$ for some $i \in\{1, \ldots, n\}$, then $\beta \in P I T L_{\mathbf{L}^{\nu_{i}} \cup C}$. To realise this, notice that changing all the superscripts of the flexible symbols in the formulas from the part of the proof which leads to $\beta$ to $\nu_{i}$ preserves its correctness. We can choose $\beta_{i}$ to be the conjunction of all the formulas from $P I T L_{\mathbf{L}^{\nu_{i} \cup C}}$ in the chosen proof of $\alpha, i=1, \ldots, n$.

Consistency in the rest of this section is with respect to $P I T L^{-}$.

4.6. The elementary diagram $\Delta$ for $M$. Here follows the precise construction of the diagram $\Delta$.

$\Delta$ is the union of the infinite ascending sequence of sets of formulas

$$
\Delta_{0} \subset \Delta_{1}^{\prime} \subset \Delta_{1} \subset \ldots \subset \Delta_{k}^{\prime} \subset \Delta_{k} \subset \ldots
$$

where $\Delta_{k}$ and $\Delta_{k+1}^{\prime}$ consist of formulas written in $\mathbf{L}_{\leq k}$ and $\mathbf{L}_{\leq k+1}^{\prime}$, respectively, for each $k<\omega . \Delta_{0}$ is a maximal consistent set with witnesses in $C_{0}^{d} \cup C_{0}^{p}$ which contains the set 
$\left\{\gamma^{\langle\rangle}: \gamma \in \Gamma\right\}$. Such a set exists by Theorem 4.5. For an arbitrary $k<\omega, \Delta_{k+1}^{\prime}$ is the extension of $\Delta_{k}$ by

the formula $\varphi^{\nu^{\prime}}$ and the formulas $\left(\square \forall\left(\chi^{\nu} \Leftrightarrow \chi^{\nu^{\prime}}\right) \wedge \ell=c ; \ell=\infty\right)$ for all $\chi$ written in $\mathbf{L}$,

for each pair of indices $\nu \in S_{\leq k}$ and $\nu^{\prime} \in S_{k+1}$ such that $\nu^{\prime}=\langle\nu, c, \varphi\rangle$ and $\left(p\left(\varphi^{\nu}\right) \neq 0 \wedge \ell=c ; \ell=\infty\right) \in \Delta_{k}$.

Lemma 4.11. If $\Delta_{k}$ is consistent, then $\Delta_{k+1}^{\prime}$ is consistent too.

The proof of this lemma is the key technical step in the entire completeness argument about our proof system for PITL.

Proof. Assume that $\Delta_{k}$ is consistent and $\Delta_{k+1}^{\prime}$ is not for the sake of contradiction. Since proofs in $P I T L^{-}$are finitary, there is a finite inconsistent $\Xi \subset \Delta_{k+1}^{\prime}$. $\Xi \nsubseteq \Delta_{k}$, because $\Delta_{k}$ is a consistent set. Hence there are finitely many $\nu^{\prime} \in S_{k+1} \backslash S_{\leq k}$ such that flexible symbols superscripted by $\nu^{\prime}$ occur in formulas from $\Xi$. These formulas are of some of the forms (4.6). Below we prove that the assumed inconsistency of $\Xi$ is preserved after withdrawing the formulas of the forms (4.6) for each such $\nu^{\prime} \in S_{k+1} \backslash S_{\leq k}$. The remaining formulas in $\Xi$ are also in $\Delta_{k}$. This will bring contradiction with the assumed consistency of $\Delta_{k}$. Let us choose one such $\nu^{\prime}$ and let $\nu^{\prime}=\langle\nu, c, \varphi\rangle$. This means that $\left(p\left(\varphi^{\nu}\right) \neq 0 \wedge \ell=c ; \ell=\infty\right) \in \Delta_{k}$. Then the formulas (4.6) for the chosen $\nu^{\prime}$ and $\nu$ are in $\Delta_{k+1}^{\prime}$. Let the formulas in $\Xi$ with flexible symbols superscripted by $\nu^{\prime}$ be $\left(\square \forall\left(\chi_{i}^{\nu} \Leftrightarrow \chi_{i}^{\nu^{\prime}}\right) \wedge \ell=c ; \ell=\infty\right), i=1, \ldots, m$, and $\varphi^{\nu^{\prime}}$. Let $\Xi_{\overline{\nu^{\prime}}}$ be the set of the remaining formulas from $\Xi$, which have no flexible symbols superscripted by $\nu^{\prime}$. Then

$$
\vdash_{\text {PITL }_{\mathbf{L}_{\leq k+1}^{\prime}}^{-}}\left(\bigwedge \Xi{\overline{\nu^{\prime}}}\right) \Rightarrow\left(\bigwedge_{i=1}^{m}\left(\square \forall\left(\chi_{i}^{\nu} \Leftrightarrow \chi_{i}^{\nu^{\prime}}\right) \wedge \ell=c ; \ell=\infty\right) \Rightarrow \neg \varphi^{\nu^{\prime}}\right) .
$$

Now Proposition 4.9 entails that

$$
\vdash_{I T L} \mathrm{t}(\alpha) \Rightarrow\left(\mathrm{t}\left(\bigwedge \Xi_{\overline{\nu^{\prime}}}\right) \Rightarrow\left(\bigwedge_{i=1}^{m}\left(\square \forall\left(\mathrm{t}\left(\chi_{i}^{\nu}\right) \Leftrightarrow \mathrm{t}\left(\chi_{i}^{\nu^{\prime}}\right)\right) \wedge \ell=c ; \ell=\infty\right) \Rightarrow \neg \mathrm{t}\left(\varphi^{\nu^{\prime}}\right)\right)\right)
$$

where $\alpha \in P I T L_{\mathbf{L}_{\leq k+1}^{\prime}}^{-}$. According to Lemma 4.10, there is a finite set of superscripts

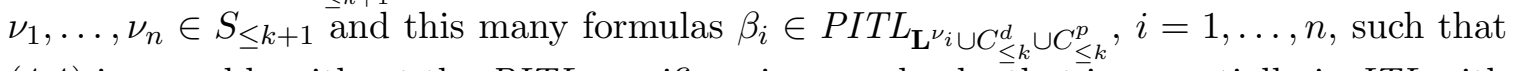
(4.4) is provable without the PITL-specific axioms and rule, that is, essentially in ITL with infinite intervals. Without loss of generality we can assume that $\beta_{1} \in P I T L_{\mathbf{L}^{\nu} \cup C_{\leq k}^{d} \cup C_{\leq k}^{p}}$ and $\beta_{2} \in P I T L_{\mathbf{L}^{\nu^{\prime}} \cup C_{\leq k}^{d} \cup C_{\leq k}^{p}}$. Then we have

$$
\begin{aligned}
& \vdash_{I T L} \mathrm{t}\left(\left(\bigwedge \Xi_{\overline{\nu^{\prime}}}\right) \wedge \bigwedge_{i=3}^{n} \square \forall \beta_{i}\right) \Rightarrow \\
& \quad\left(\mathrm{t}\left(\square \forall \beta_{1}\right) \wedge \mathrm{t}\left(\square \forall \beta_{2}\right) \wedge \bigwedge_{i=1}^{m}\left(\square \forall\left(\mathrm{t}\left(\chi_{i}^{\nu}\right) \Leftrightarrow \mathrm{t}\left(\chi_{i}^{\nu^{\prime}}\right)\right) \wedge \ell=c ; \ell=\infty\right) \Rightarrow \neg \mathrm{t}\left(\varphi^{\nu^{\prime}}\right)\right) .
\end{aligned}
$$

All the flexible symbols on the right of the main $\Rightarrow$ in this formula except $\ell$ are superscripted by either $\nu$ or $\nu^{\prime}$ and the superscript $\nu^{\prime}$ does not appear on symbols in the formula on the left of $\Rightarrow$. Hence by Craig interpolation (Theorem 4.2) some ITL formula $\lambda$ written in 
$\left(\mathbf{L}^{\nu} \cup C_{\leq k}^{d} \cup C_{\leq k}^{p}\right)_{I T L}$ satisfies both

$$
\vdash_{I T L} \mathrm{t}\left(\left(\bigwedge \Xi_{\overline{\nu^{\prime}}}\right) \wedge \bigwedge_{i=3}^{n} \square \forall \beta_{i}\right) \Rightarrow \lambda
$$

and

$$
\vdash_{I T L} \bigwedge_{i=1}^{m}\left(\square \forall\left(\mathrm{t}\left(\chi_{i}^{\nu}\right) \Leftrightarrow \mathrm{t}\left(\chi_{i}^{\nu^{\prime}}\right)\right) \wedge \ell=c ; \ell=\infty\right) \Rightarrow\left(\left(\lambda \wedge \mathrm{t}\left(\square \forall \beta_{1}\right)\right) \Rightarrow\left(\mathrm{t}\left(\square \forall \beta_{2}\right) \Rightarrow \neg \mathrm{t}\left(\varphi^{\nu^{\prime}}\right)\right)\right) .
$$

The formulas $\lambda \wedge \mathrm{t}\left(\square \forall \beta_{1}\right)$ and $\mathrm{t}\left(\square \forall \beta_{2}\right) \Rightarrow \neg \mathrm{t}\left(\varphi^{\nu^{\prime}}\right)$ in (4.8) are written in $\left(\mathbf{L}^{\nu} \cup C_{\leq k}^{d} \cup C_{\leq k}^{p}\right)$ ITL and $\left(\mathbf{L}^{\nu^{\prime}} \cup C_{\leq k}^{d} \cup C_{\leq k}^{p}\right)_{I T L}$, respectively. A bijection can be defined between the sets of the flexible symbols of these two vocabularies, excluding $\ell$, in which the flexible symbol $s^{\prime} \in\left(\mathbf{L}^{\nu^{\prime}} \cup C_{\leq k}^{d} \cup C_{\leq k}^{p}\right)_{I T L} \backslash\{\ell\}$ which corresponds to $s \in\left(\mathbf{L}^{\nu} \cup C_{\leq k}^{d} \cup C_{\leq k}^{p}\right)_{I T L} \backslash\{\ell\}$ is obtained by changing all the superscripts $\nu$ in $s$ to $\nu^{\prime}$ and vice-versa. If $s$ is of the form $\mathrm{p}_{\mathrm{t}(\psi)}$ (see Definition 4.6), it may have more than one occurrence of a superscript $\nu$ in the subscript formula $\mathrm{t}(\psi)$. All these occurrences have to be changed. This bijection allows us to apply interval-related interpolation (Theorem 4.1) to (4.8) and conclude that some $I T L$ formulas $\theta_{I T L} \in\left(\mathbf{L}^{\nu} \cup C_{\leq k}^{d} \cup C_{\leq k}^{p}\right)_{I T L}$ and $\theta_{I T L}^{\prime} \in\left(\mathbf{L}^{\nu^{\prime}} \cup C_{\leq k}^{d} \cup C_{\leq k}^{p}\right)_{I T L}$ which can be obtained from each other by replacing the corresponding flexible symbols from their respective vocabularies satisfy

$$
\vdash_{I T L} \lambda \wedge \mathrm{t}\left(\square \forall \beta_{1}\right) \wedge c<\infty \wedge \ell=\infty \Rightarrow\left(\ell=c \wedge \theta_{I T L} ; \ell=\infty\right)
$$

and

$$
\vdash_{I T L}\left(\ell=c \wedge \theta_{I T L}^{\prime} ; \ell=\infty\right) \Rightarrow\left(\mathrm{t}\left(\square \forall \beta_{2}\right) \Rightarrow \neg \mathrm{t}\left(\varphi^{\nu^{\prime}}\right)\right)
$$

which by simply changing all superscripts $\nu^{\prime}$ to $\nu$ implies

$$
\vdash_{I T L}\left(\ell=c \wedge \theta_{I T L} ; \ell=\infty\right) \Rightarrow\left(\mathrm{t}\left(\square \forall \beta_{2}^{\prime}\right) \Rightarrow \neg \mathrm{t}\left(\varphi^{\nu}\right)\right)
$$

where $\beta_{2}^{\prime}$ is the result of changing all the superscripts $\nu^{\prime}$ of the flexible symbols in $\beta_{2}$ to $\nu$. By (4.7) and (4.9) we obtain

$$
\vdash_{I T L} \mathrm{t}\left(\left(\bigwedge \Xi_{\overline{\nu^{\prime}}}\right) \wedge \bigwedge_{i=3}^{n} \square \forall \beta_{i}\right) \wedge \mathrm{t}\left(\square \forall \beta_{1}\right) \wedge c<\infty \wedge \ell=\infty \Rightarrow\left(\ell=c \wedge \theta_{I T L} ; \ell=\infty\right)
$$

The formula $\theta_{I T L}$ is the t-translation of some PITL formula written in $\mathbf{L}^{\nu} \cup C_{\leq k}^{d} \cup C_{\leq k}^{p}$ which, in its turn, has the form $\theta^{\nu}$ where $\theta$ is a formula written in $\mathbf{L} \cup C_{\leq k}^{d} \cup C_{\leq k}^{p}$. (Then $\theta_{I T L}^{\prime}$ is $\mathrm{t}\left(\theta^{\nu^{\prime}}\right)$.) Hence we have

$$
\vdash_{P I T L_{\mathbf{L}_{\leq k+1}^{\prime}}^{-}}\left(\bigwedge \Xi_{\overline{\nu^{\prime}}}\right) \wedge \bigwedge_{i=3}^{n} \square \forall \beta_{i} \wedge \square \forall \beta_{1} \wedge c<\infty \wedge \ell=\infty \Rightarrow\left(\ell=c \wedge \theta^{\nu} ; \ell=\infty\right) .
$$

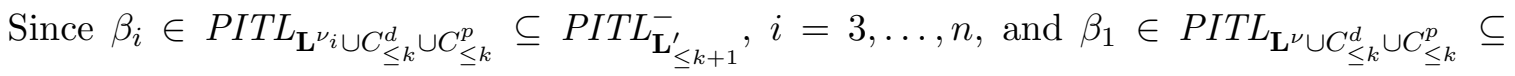
$P I T L_{\mathbf{L}_{\leq k+1}^{\prime}}^{-}$, the above formula can be simplified to

$$
\vdash_{P I T L_{\mathbf{L}^{\prime} k+1}^{-}}\left(\bigwedge \Xi_{\overline{\nu^{\prime}}}\right) \wedge c<\infty \wedge \ell=\infty \Rightarrow\left(\ell=c \wedge \theta^{\nu} ; \ell=\infty\right) .
$$


Since $\left(p\left(\varphi^{\nu}\right) \neq 0 \wedge \ell=c ; \ell=\infty\right) \in \Delta_{k}, c<\infty, \ell=\infty \in \Delta_{k}$ too. This implies that $\left(\ell=c \wedge \theta^{\nu} ; \ell=\infty\right) \in C n_{\mathbf{L}_{\leq k+1}^{\prime}}\left(\Delta_{k} \cup \Xi_{\overline{\nu^{\prime}}}\right)$. Similarly, (4.10) implies than

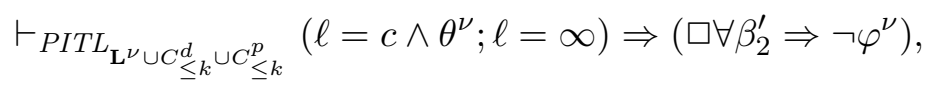

and, since $\beta_{2}^{\prime}$ is a PITL theorem written in the vocabulary $\mathbf{L}^{\nu} \cup C_{\leq k}^{d} \cup C_{\leq k}^{p}$,

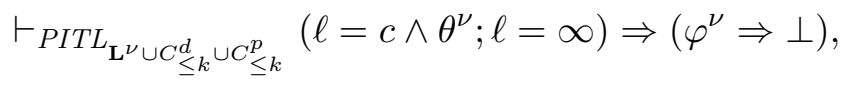

Now by an application of the rule $P_{\leq}$to (4.12), where the flexible symbols have no other superscript except $\nu$ as required by our restricted way of applying this $P I T L$-specific rule, we obtain

which implies

$$
\vdash_{\text {PITL }_{\mathbf{L}_{\leq k+1}^{\prime}}^{-}} \ell=c \wedge \theta^{\nu} \wedge \ell<\infty \Rightarrow p\left(\varphi^{\nu}\right) \leq p(\perp)
$$

by $P_{\perp}$ and, finally,

$$
\vdash_{P^{\prime T L} L_{\mathbf{L}_{\leq k+1}^{\prime}}^{-}} \ell=c \wedge \theta^{\nu} \wedge \ell<\infty \Rightarrow p\left(\varphi^{\nu}\right)=0
$$

$$
\vdash_{P I T L_{\mathbf{L}^{\prime} k+1}^{-}}\left(\ell=c \wedge \theta^{\nu} \wedge \ell<\infty ; \ell=\infty\right) \Rightarrow\left(p\left(\varphi^{\nu}\right)=0 \wedge \ell=c ; \ell=\infty\right)
$$

by an application of the ITL proof rule Mono. Since $c<\infty,\left(\ell=c \wedge \theta^{\nu} ; \ell=\infty\right) \in$ $C n_{\mathbf{L}_{\leq k+1}^{\prime}}\left(\Delta_{k} \cup \Xi_{\overline{\nu^{\prime}}}\right)$, this implies $\left(p\left(\varphi^{\nu}\right)=0 \wedge \ell=c ; \ell=\infty\right) \in C n_{\mathbf{L}_{\leq k+1}^{\prime}}\left(\Delta_{k} \cup \Xi_{\overline{\nu^{\prime}}}\right)$. Hence $\Delta_{k} \cup \Xi_{\overline{\nu^{\prime}}}$ is just as inconsistent as $\Delta_{k} \cup \Xi$, because the reason for all the formulas with flexible symbols superscripted by $\nu^{\prime}=\langle\nu, c, \varphi\rangle$ to be in the finite subset $\Xi$ of $\Delta_{k+1}^{\prime}$ is $\left(p\left(\varphi^{\nu}\right) \neq 0 \wedge \ell=c ; \ell=\infty\right) \in \Delta_{k}$. We can continue by showing that taking away the formulas of the form (4.6) for some other superscript $\nu^{\prime \prime} \in S_{k+1} \backslash S_{\leq k}$ leads to a subset $\left(\Xi \overline{\nu^{\prime}}\right) \overline{\nu^{\prime \prime}}$ of $\Xi_{\overline{\nu^{\prime}}}$ such that $\Delta_{k} \cup\left(\Xi_{\overline{\nu^{\prime}}}\right)_{\overline{\nu^{\prime \prime}}}$ is still inconsistent, etc., until there are no more symbols with superscripts from $S_{k+1} \backslash S_{\leq k}$ in the remaining subset of $\Xi$, which then will be a subset of $\Delta_{k}$. This is the sought contradiction, because we assume that $\Delta_{k}$ is consistent.

For an arbitrary $k<\omega$, if $\Delta_{k+1}^{\prime}$ is consistent, then $\Delta_{k+1}$ is defined as some maximal consistent set which contains $\Delta_{k+1}^{\prime}$ and has witnesses in $C_{k+1}^{d} \cup C_{k+1}^{p}$. Its existence follows from Theorem 4.5 again. Then Lemma 4.11 implies that all the sets in the sequence (4.5) are consistent. Furthermore, obviously $\Delta$ is a maximal consistent set in $\mathbf{L}_{D}$ with respect to $\vdash_{\text {PITL }^{-}}$and has witnesses in $C^{d} \cup C^{p}$. The construction of $\Delta$ is complete.

4.7. The PITL model $M$. Since $\Delta$ is a maximal consistent set of PITL formulas written in $\mathbf{L}_{D}$ with witnesses in $C^{d} \cup C^{p}, \mathrm{t}(\Delta)$ is maximal consistent set of $I T L$ formulas written in $\left(\mathbf{L}_{D}\right)_{I T L}$ with witnesses in $C^{d} \cup C^{p}$ too. We use this to construct the model $M$ at two steps, the first being the construction of a canonical ITL model $M_{I T L}$ which satisfies $\mathrm{t}(\Delta)$ and the second being the construction of $M$ itself. This way we avoid the repetition of the non- $P I T L$-specific steps in the construction of $M$ which are as in [WX04]. 


\subsubsection{The ITL counterpart of $M$. Let}

$$
c_{1} \equiv c_{2} \text { iff } c_{1}=c_{2} \in \Delta
$$

for constants $c_{1}, c_{2} \in C^{d}$ and $c_{1}, c_{2} \in C^{p}$. Clearly, $\equiv$ is an equivalence relation on the constants from $C^{d} \cup C^{p}$. Let $[c]$ denote the $\equiv$-equivalence class which contains $c$ for each $c \in C^{d} \cup C^{p}$. Let

$$
T=\left\{[c]: c \in C^{d}\right\}, D=T \text {, and } U=\left\{[c]: c \in C^{d}\right\} .
$$

Let

$$
\left[c^{\prime}\right] \leq\left[c^{\prime \prime}\right] \text { iff } c^{\prime} \leq c^{\prime \prime} \in \Delta
$$

for $c^{\prime}, c^{\prime \prime} \in C^{d}$. Clearly, $\leq$ is a linear ordering on $T$. Let $c_{\infty}$ be a witness in $C^{d}$ for the formula $\exists x(x=\infty)$ in $\Delta$. Then clearly $\left\langle T, \leq,\left[c_{\infty}\right]\right\rangle$ is a time domain.

Given $\left[\left[c^{\prime}\right],\left[c^{\prime \prime}\right]\right] \in \tilde{\mathbf{I}}(T)$, we denote the set of formulas written in $\mathbf{L}_{D}$

$$
\left\{\varphi:\left(\left(\ell=c^{\prime} ; \varphi\right) \wedge \ell=c^{\prime \prime} ; \top\right) \vee\left(c^{\prime \prime}=\infty \wedge\left(\ell=c^{\prime} ; \varphi\right)\right) \in \Delta\right\}
$$

by $\Delta_{\left[\left[c^{\prime}\right],\left[c^{\prime \prime}\right]\right]}$. To understand the definition of $\Delta_{\left[\left[c^{\prime}\right],\left[c^{\prime \prime}\right]\right]}$, recall our choice to start from a set $\Gamma$ such that $\ell=\infty \in \Gamma$ and, consequently, $\ell=\infty \in \Delta$. Let $c_{0} \in C^{d}$ be a witness for $\exists x(x=0)$ in $\Delta$ and $\sigma_{0}=\left[\left[c_{0}\right],\left[c_{\infty}\right]\right]$ for the rest of the section. Then obviously $\Delta_{\sigma_{0}}=\Delta$ and

for all $\varphi \in \mathbf{L}_{D}$.

$$
\varphi \in \Delta_{\left[\left[c^{\prime}\right],\left[c^{\prime \prime}\right]\right]} \text { iff }\left(\ell=c^{\prime} ; \varphi\right) \in \Delta_{\left[\left[c_{0}\right],\left[c^{\prime \prime}\right]\right]}
$$

We define the mapping $I_{I T L}$ of $\left(\mathbf{L}_{D}\right)_{\text {ITL }}$ by the clauses:

$I_{I T L}(x), I_{I T L}(d) \in A$ for individual variables $x$ and constants $d$ where $A=D$ for $x$ and $d$ of the duration sort and $A=U$ otherwise, and

$$
I_{I T L}(x)=\left\{c \in C^{d} \cup C^{p}: c=x \in \mathrm{t}(\Delta)\right\}, I_{I T L}(d)=\left\{c \in C^{d} \cup C^{p}: c=d \in \mathrm{t}(\Delta)\right\} .
$$

$I_{I T L}(f): A_{1} \times \ldots \times A_{\# f} \rightarrow A_{\# f+1}$ rigid function symbols $f$ where $A_{1}, \ldots, A_{\# f+1}$ are either $D$ or $U$, depending on the sort of the respective arguments of $f$ and the sort of its value, and

$$
I_{I T L}(f)\left(\left[c_{1}\right], \ldots,\left[c_{\# f}\right]\right)=\left\{c \in C^{d} \cup C^{p}: c=f\left(c_{1}, \ldots, c_{\# f}\right) \in \mathrm{t}(\Delta)\right\} .
$$

$I_{\text {ITL }}(R): A_{1} \times \ldots \times A_{\# R} \rightarrow\{0,1\}$ for rigid relation symbols $R$ where $A_{1}, \ldots, A_{\# R}$ are as for function symbols, and

$$
I_{\text {ITL }}(R)\left(\left[c_{1}\right], \ldots,\left[c_{\# R}\right]\right)=1 \text { iff } R\left(c_{1}, \ldots, c_{n}\right) \in \mathrm{t}(\Delta) .
$$

$I_{I T L}(d): \tilde{\mathbf{I}}(T) \rightarrow A, I_{I T L}(f): \tilde{\mathbf{I}}(T) \times A_{1} \times \ldots \times A_{\# f} \rightarrow A_{\# f+1}$ and $I_{\text {ITL }}(R): A_{1} \times \ldots \times A_{\# R} \rightarrow\{0,1\}$ for flexible $d, f$ and $R$, respectively, where the $A$ s are as for rigid symbols.

Similarly,

$$
I_{I T L}(d)(\sigma)=\left\{c \in C^{d} \cup C^{p}: c=d \in \mathrm{t}\left(\Delta_{\sigma}\right)\right\} .
$$

$$
I_{I T L}(f)\left(\sigma,\left[c_{1}\right], \ldots,\left[c_{\# f}\right]\right)=\left\{c \in C^{d} \cup C^{p}: c=f\left(c_{1}, \ldots, c_{\# f}\right) \in \mathrm{t}\left(\Delta_{\sigma}\right)\right\} .
$$

Finally, $I_{I T L}(R)\left(\sigma,\left[c_{1}\right], \ldots,\left[c_{\# R}\right]\right)=1$ iff $R\left(c_{1}, \ldots, c_{\# R}\right) \in \mathrm{t}\left(\Delta_{\sigma}\right)$. 
A lengthy but otherwise straighforward argument, which is standard for canonical models, shows that the above definitions are correct, $\left\langle D, I_{I T L}(+), I_{I T L}(0), I_{I T L}(\infty)\right\rangle$ is a duration domain, $\left\langle U, I_{I T L}(+), I_{I T L}(0), I_{I T L}(1)\right\rangle$ is a probability domain and $I_{I T L}(\ell)$ is a measure function from $\tilde{\mathbf{I}}(T)$ to $D$,

$$
F=\left\langle\left\langle T, \leq, I_{I T L}(\infty)\right\rangle,\left\langle D, I_{I T L}(+), I_{I T L}(0), I_{I T L}(\infty)\right\rangle,\left\langle U, I_{I T L}(+), I_{I T L}(0), I_{I T L}(1)\right\rangle, I(\ell)\right\rangle
$$

is a two-sorted frame for $I T L$ with infinite intervals and $I$ is an $I T L$ interpretation of $\left(\mathbf{L}_{D}\right)_{I T L}$ into $F$, which means that $M_{I T L}=\left\langle F, I_{I T L}\right\rangle$ is a two-sorted ITL model for $\left(\mathbf{L}_{D}\right)_{I T L}$. The standard truth lemma holds for $M_{I T L}$, which is a canonical model:

Lemma 4.12 (Truth Lemma for $\left.M_{I T L}\right)$. Let $\sigma \in \tilde{\mathbf{I}}(T)$. Then

$$
\left(I_{I T L}\right)_{\sigma}(t)=\left\{c \in C^{d} \cup C^{p}: t=c \in \mathrm{t}\left(\Delta_{\sigma}\right)\right\} \text { and } M_{I T L}, \sigma \models \varphi \text { iff } \varphi \in \mathrm{t}\left(\Delta_{\sigma}\right)
$$

for every term $t$ and every formula $\varphi$ written in the vocabulary $\left(\mathbf{L}_{D}\right)_{\text {ITL }}$.

4.7.2. The model $M$. Our next step is to define the PITL model $M=\langle F, \mathbf{W}, I, P\rangle$ itself. The vocabulary of $M$ is $\mathbf{L} \cup C^{d} \cup C^{p}$ and its frame is $F$. Let $\Pi$ denote the set of the functions $\pi: V \rightarrow D \cup U$ where $V$ is a finite set of individual variables in $\mathbf{L}$ and $\pi(x)$ is in the domain which corresponds to the sort of $x$ for each $x \in V$. We define $\mathbf{W}$ as the set $S \times \Pi$. Given $\nu \in S$, we define the interpretation $I_{\nu}$ by the equalities

$$
I_{\nu}(s)=I_{I T L}(s)
$$

for rigid $s \in \mathbf{L} \cup C^{d} \cup C^{p}$, including the individual variables,

$$
I_{\nu}(\ell)=m \text { and } I_{\nu}(d)=I_{I T L}\left(d^{\nu}\right)
$$

for flexible constants $d \in \mathbf{L} \backslash\{\ell\}$ and

$$
I_{\nu}(s)\left(\sigma, a_{1}, \ldots, a_{\# s}\right)=I_{I T L}\left(s^{\nu}\right)\left(\sigma, a_{1}, \ldots, a_{\# s}\right)
$$

for other flexible $s \in \mathbf{L}$. Now $\mathbf{W}$ consists of all the variants of the $I_{\nu}$ for all $\nu \in S$.

Given $w=\langle\nu, \pi\rangle$ such that $\operatorname{dom} \pi=\left\{x_{1}, \ldots, x_{n}\right\}$, we put

$$
I^{w}=\left(I_{\nu}\right)_{x_{1},, \ldots, x_{n}}^{\pi\left(x_{1}\right), \ldots, \pi\left(x_{n}\right)} .
$$

Some auxiliary notation is needed for the definition of $P^{w}$.

Let $\varphi$ be a formula written in $\mathbf{L} \cup C^{d} \cup C^{p}, F V(\varphi)=\emptyset, \nu \in S$ and $\left[\left[c^{\prime}\right],\left[c^{\prime \prime}\right]\right] \in \tilde{I}(T)$. Then we denote the set

$\left\{\nu^{\prime} \in S: \varphi^{\nu^{\prime}} \in \Delta_{\left[\left[c^{\prime}\right],\left[c_{\infty}\right]\right]},\left(\square \forall\left(\chi^{\nu} \Leftrightarrow \chi^{\nu^{\prime}}\right) \wedge \ell=\left[c^{\prime \prime}\right] ; \top\right) \in \Delta_{\left[\left[c^{\prime}\right],\left[c_{\infty}\right]\right]}\right.$ for all $\chi$ in $\left.\mathbf{L} \cup C^{d} \cup C^{p}\right\}$ by $S_{\varphi, \nu,\left[\left[c^{\prime},\left[c^{\prime \prime}\right]\right]\right.}$. We use $S_{\varphi, \nu,\left[\left[c^{\prime}\right],\left[c^{\prime \prime}\right]\right]}$ to define a syntactical conterpart $(()$.$) to \llbracket . \rrbracket$ in our model under construction. If $\psi$ is a formula written in $\mathbf{L} \cup C^{d} \cup C^{p}, F V(\psi)=\left\{x_{1}, \ldots, x_{n}\right\}$ and $c_{i} \in I^{\langle\nu, \pi\rangle}\left(x_{i}\right), i=1, \ldots, n$, then we put

$$
((\varphi))_{\langle\nu, \pi\rangle,\left[\left[c^{\prime}\right],\left[c^{\prime \prime}\right]\right]}=\left\{\left\langle\nu^{\prime}, \pi^{\prime}\right\rangle \in \mathbf{W}: \nu^{\prime} \in S_{\left[c_{1} / x_{1}, \ldots, c_{n} / x_{n}\right] \varphi, \nu,\left[\left[c^{\prime}\right],\left[c^{\prime \prime}\right]\right]}, \pi^{\prime} \in \Pi\right\} .
$$

Clearly, the set on the right of $=$ in (4.14) does not depend on the precise choice of $c_{i} \in$ $I^{\langle\nu, \pi\rangle}\left(x_{i}\right), i=1, \ldots, n$. The truth lemma about $M$ which is proved below entails that

Note that

$$
((\varphi))_{w,\left[\left[c^{\prime}\right],\left[c^{\prime \prime}\right]\right]}=\llbracket \varphi \rrbracket_{M, w,\left[\left[c^{\prime}\right],\left[c^{\prime \prime}\right]\right]}
$$

$$
((\varphi))_{w,\left[\left[c^{\prime}\right],\left[c^{\prime \prime}\right]\right]}=\left(\left(\left(\ell=c^{\prime} ; \varphi\right)\right)\right)_{w,\left[\left[c_{0}\right],\left[c^{\prime \prime}\right]\right]}
$$


follows from (4.13) and therefore the rest of the construction steps involve mostly intervals $\sigma \in \tilde{I}(T)$ such that $\min \sigma=\left[c_{0}\right]$. Given $w \in \mathbf{W}, w=\langle\nu, \pi\rangle$, a formula $\varphi$ written in $\mathbf{L} \cup C^{d} \cup C^{p}$ whose free variables are $x_{1}, \ldots, x_{n}, \nu \in S, c_{i} \in I^{w}\left(x_{i}\right), i=1, \ldots, n$, and $\left[c^{\prime \prime}\right] \in T$ we define $P^{w}$ on the subsets of $\mathbf{W}$ of the form (4.14) by the equality

$$
P^{w}\left(\left[c^{\prime \prime}\right],((\varphi))_{w,\left[\left[c_{0}\right],\left[c^{\prime \prime}\right]\right]}\right)=\left\{c \in C^{p}: p\left(\left[c_{1} / x_{1}, \ldots, c_{n} / x_{n}\right] \varphi^{\nu}\right)=c \in \Delta_{\left[\left[c_{0}\right],\left[c^{\prime \prime}\right]\right]}\right\} .
$$

For this definition to be correct, we need to have

$$
p\left(\left[c_{1} / x_{1}, \ldots, c_{n} / x_{n}\right] \varphi^{\nu}\right)=c \in \Delta_{\left[\left[c_{0}\right],\left[c^{\prime \prime}\right]\right]} \text { iff } p\left(\left[c_{1} / x_{1}, \ldots, c_{n} / x_{n}\right] \psi^{\nu}\right)=c \in \Delta_{\left[\left[c_{0}\right],\left[c^{\prime \prime}\right]\right]}
$$

for formulas $\varphi$ and $\psi$ such that

$$
((\varphi))_{w,\left[\left[c_{0}\right],\left[c^{\prime \prime}\right]\right]}=((\psi))_{w,\left[\left[c_{0}\right],\left[c^{\prime \prime}\right]\right]}
$$

and $c_{i} \in I^{w}\left(x_{i}\right), i=1, \ldots, n$, where $\left\{x_{1}, \ldots, x_{n}\right\}=F V(\varphi) \cup F V(\psi)$. To prove it, assume that

$$
p\left(\left[c_{1} / x_{1}, \ldots, c_{n} / x_{n}\right] \varphi^{\nu}\right)<p\left(\left[c_{1} / x_{1}, \ldots, c_{n} / x_{n}\right] \psi^{\nu}\right) \in \Delta_{\left[\left[c_{0}\right],\left[c^{\prime \prime}\right]\right]}
$$

for the sake of contradiction. Then

$$
p\left(\left[c_{1} / x_{1}, \ldots, c_{n} / x_{n}\right]\left(\psi^{\nu} \wedge \neg \varphi^{\nu}\right)\right) \neq 0 \in \Delta_{\left[\left[c_{0}\right],\left[c^{\prime \prime}\right]\right]}
$$

by PITL3 from Section 3.2 . If $c^{\prime \prime}<\infty \in \Delta$, then this implies that

$$
\left\langle\left\langle\nu, c^{\prime \prime}, \psi \wedge \neg \varphi\right\rangle, \pi^{\prime}\right\rangle \in((\psi))_{w,\left[\left[c_{0}\right],\left[c^{\prime \prime}\right]\right]} \backslash((\varphi))_{w,\left[\left[c_{0}\right],\left[c^{\prime \prime}\right]\right]}
$$

where $\operatorname{dom} \pi^{\prime}=F V(\varphi) \cup F V(\psi)$ and $\pi^{\prime}\left(x_{i}\right)=I^{w}\left(x_{i}\right) . i=1, \ldots, n$, which contradicts (4.17). If $c^{\prime \prime}=\infty \in \Delta$, then the appropriate instances of $P_{\infty}$ and PITL2 from Section 3.2 imply that

and, consequently,

$$
p\left(\left[c_{1} / x_{1}, \ldots, c_{n} / x_{n}\right]\left(\psi^{\nu} \wedge \neg \varphi^{\nu}\right)\right)=1 \in \Delta_{\left[\left[c_{0}\right],\left[c^{\prime \prime}\right]\right]}
$$

$$
\left[c_{1} / x_{1}, \ldots, c_{n} / x_{n}\right]\left(\psi^{\nu} \wedge \neg \varphi^{\nu}\right) \in \Delta_{\left[\left[c_{0}\right],\left[c^{\prime \prime}\right]\right]} .
$$

This implies that $w$ itself is in $((\psi))_{w,\left[\left[c_{0}\right],\left[c^{\prime \prime}\right]\right]} \backslash((\varphi))_{w,\left[\left[c_{0}\right],\left[c^{\prime \prime}\right]\right]}$, which contradicts (4.17) too.

The presence of all the instances of $P_{\perp}, P_{\top}$ and $P_{+}$written in the vocabularies $\mathbf{L}^{\nu} \cup$ $C^{d} \cup C^{p}, \nu \in S$, in $\Delta_{\left[\left[c_{0}\right],\left[c^{\prime \prime}\right]\right]}$ implies that $\lambda X . P^{w}\left(\left[c^{\prime \prime}\right], X\right)$ is a finitely additive probability function on the boolean algebra

$$
\left\langle\left\{((\psi))_{w,\left[\left[c_{0}\right],\left[c^{\prime \prime}\right]\right]}: \psi \in \mathbf{L}\right\}, \cap, \cup, \emptyset, \mathbf{W}_{w,\left[c^{\prime \prime}\right]}\right\rangle
$$

for every $w \in \mathbf{W}$ and every $\left[c^{\prime \prime}\right] \in T$. Note that this algebra contains the sets $((\psi))_{w,\left[\left[c^{\prime}\right],\left[c^{\prime \prime}\right]\right]}$ for all $c^{\prime} \in C^{d}$ such that $c^{\prime} \leq c^{\prime \prime} \in \Delta$ because of (4.16). Clearly, $M=\langle F, \mathbf{W}, I, P\rangle$ is a $P I T L$ model for the vocabulary $\mathbf{L} \cup C^{d} \cup C^{p}$.

Obviously if $w=\langle\nu, \pi\rangle$ for some $\pi \in \Pi$ then $\left\{\left\langle\langle\nu, c, \varphi\rangle, \pi^{\prime}\right\rangle: \pi^{\prime} \in \Pi\right\} \subseteq \in \mathbf{W}_{w,[c]}$ for all $\nu \in S_{\leq k}, c \in C^{d}$ and all $\varphi$ written in $\mathbf{L}_{\leq k}$ such that $\left(p\left(\varphi^{\nu}\right) \neq 0 \wedge \ell=c ; \top\right) \in \Delta$ and all $k<\omega$, because, according to the construction of $\Delta$, in this case

$$
\left(\square \forall\left(\chi^{\nu} \Leftrightarrow \chi^{\langle\nu, c, \varphi\rangle}\right) \wedge \ell=c ; \top\right) \in \Delta
$$

for all formulas $\chi$ written in $\mathbf{L} \cup C^{d} \cup C^{p}$, and in particular for $\chi$ of the forms $d=x$, $f\left(x_{1}, \ldots, x_{\# f}\right)=x_{\# f+1}, R\left(x_{1}, \ldots, x_{\# R}\right)$ and $p(\psi)=x$ where $d, f$ and $R$ are flexible constants, function and relation symbols from $\mathbf{L}$, and $\psi$ is written in $\mathbf{L} \cup C^{d} \cup C^{p}$ respectively. Furthermore, if $I^{w}$ is a variant of $I^{v}$ and $P^{w}=P^{v}$ for some $w, v \in \mathbf{W}$, then $\mathbf{W}_{w,[c]}=\mathbf{W}_{v,[c]}$ for all $[c] \in T$.

Here follows the truth lemma for $M$ : 
Lemma 4.13 (Truth Lemma for $M)$. Let $\sigma \in \tilde{\mathbf{I}}(T), w \in \mathbf{W}$ and $w=\langle\nu, \pi\rangle$. If $t$ is a term written in $\mathbf{L}_{D}, F V(t)=\left\{x_{1}, \ldots, x_{n}\right\}$ and $c_{1}, \ldots, c_{n} \in C^{d} \cup C^{p}$ are such that $c_{i} \in I^{w}\left(x_{i}\right)$, $i=1, \ldots, n$, then

$$
w_{\sigma}(t)=\left\{c \in C^{d} \cup C^{p}:\left[c_{1} / x_{1}, \ldots, c_{n} / x_{n}\right] t^{\nu}=c \in \Delta_{\sigma}\right\} .
$$

If $\varphi$ is a formula written in $\mathbf{L}_{D}, F V(\varphi)=\left\{x_{1}, \ldots, x_{n}\right\}$ and $c_{1}, \ldots, c_{n}$ satisfy the same conditions as above, then

$$
M, w, \sigma \models \varphi \text { iff }\left[c_{1} / x_{1}, \ldots, c_{n} / x_{n}\right] \varphi^{\nu} \in \Delta_{\sigma} .
$$

We use the constants $c_{1}, \ldots, c_{n}$ in the formulation of the lemma, because we need it to apply to $w \in \mathbf{W}$ with variants to some interpretation of the form $I_{\nu}$, and not just to the interpretations $I_{\nu}, \nu \in S$, themselves.

Proof. The proof is by simultaneous induction on the length of terms and formulas. The clause of the lemma about formulas implies (4.15).

The induction base and the steps for formulas and for terms built using constants, variables and function symbols are as in (non-probabilistic) ITL and we omit them. We only do the case of probabilistic terms $p(\psi)$. According to our definition, $F V(p(\psi))=F V(\psi)$. Let $x_{1}, \ldots, x_{n}$ and $c_{1}, \ldots, c_{n}$ be as in the lemma and $\sigma=\left[\left[c^{\prime}\right],\left[c^{\prime \prime}\right]\right]$. Since

$$
\begin{aligned}
w_{\left[\left[c^{\prime}\right],\left[c^{\prime \prime}\right]\right]}(p(\psi)) & =P^{w}\left(\left[c^{\prime \prime}\right], \llbracket \psi \rrbracket_{M, w,\left[\left[c^{\prime}\right],\left[c^{\prime \prime}\right]\right]}\right) \\
& =P^{w}\left(\left[c^{\prime \prime}\right], \llbracket\left(\ell=c^{\prime} ; \psi\right) \rrbracket_{M, w,\left[\left[c_{0}\right],\left[c^{\prime \prime}\right]\right]}\right) \\
& =w_{\left[\left[c_{0}\right],\left[c^{\prime \prime}\right]\right]}\left(p\left(\left(\ell=c^{\prime} ; \psi\right)\right)\right)
\end{aligned}
$$

and

$$
\left[c_{1} / x_{1}, \ldots, c_{n} / x_{n}\right] p\left(\psi^{\nu}\right)=c \in \Delta_{\left[\left[c^{\prime}\right],\left[c^{\prime \prime}\right]\right]} \text { iff }\left[c_{1} / x_{1}, \ldots, c_{n} / x_{n}\right] p\left(\left(\ell=c^{\prime} ; \psi^{\nu}\right)\right)=c \in \Delta_{\left[\left[c_{0}\right],\left[c^{\prime \prime}\right]\right]}
$$

because of the instances $\left(\ell=c^{\prime} ; p(\psi)=d\right) \Rightarrow p\left(\left(\ell=c^{\prime} ; \psi\right)\right)=d$ of $P_{;}$, which are in $\Delta_{\left[c_{0}\right],\left[c^{\prime \prime}\right]}$ for all $d \in C^{p}$, it is sufficient to prove

$w_{\left[\left[c_{0}\right],\left[c^{\prime \prime}\right]\right]}\left(p\left(\left(\ell=c^{\prime} ; \psi\right)\right)\right)=\left\{c \in C^{d} \cup C^{p}: p\left(\left(\ell=c^{\prime} ;\left[c_{1} / x_{1}, \ldots, c_{n} / x_{n}\right] \psi^{\nu}\right)\right)=c \in \Delta_{\left[\left[c_{0}\right],\left[c^{\prime \prime}\right]\right]}\right\}$.

By the induction hypothesis, the lemma holds for $\psi$ and therefore

$$
((\psi))_{w,\left[\left[c^{\prime}\right],\left[c^{\prime \prime}\right]\right]}=\llbracket \psi \rrbracket_{M, w,\left[\left[c^{\prime}\right],\left[c^{\prime \prime}\right]\right]},
$$

which implies

$$
\left(\left(\left(\ell=c^{\prime} ; \psi\right)\right)\right)_{w,\left[\left[c_{0}\right],\left[c^{\prime \prime}\right]\right]}=\llbracket\left(\ell=c^{\prime} ; \psi\right) \rrbracket_{M, w,\left[\left[c_{0}\right],\left[c^{\prime \prime}\right]\right]}
$$

by (4.16) and the definition of $\llbracket \cdot \rrbracket_{M, w,\left[.,\left[c^{\prime \prime}\right]\right]}$. Now (4.18) follows from the definition of $P^{w}$.

We conclude the presentation of $M$ with the observation that $S$ and the domains in $F$ are countably-infinite and therefore every interpretation in $\mathbf{W}$ has only countably many variants, which entails that $\mathbf{W}$ is a countably-infinite set.

4.8. The completeness theorem. Now it is easy to prove the strong completeness theorem for our proof system for PITL.

Theorem 4.14. Let $\mathbf{L}$ be a PITL vocabulary and $\Gamma$ be a set of formulas written in $\mathbf{L}$ which is consistent with the proof system from Section 3. Then there exists a model $M_{\Gamma}=$ $\left\langle F_{\Gamma}, \mathbf{W}_{\Gamma}, I_{\Gamma}, P_{\Gamma}\right\rangle$ for $\mathbf{L}$ and an $w_{0} \in \mathbf{W}_{\Gamma}$ and a time interval $\sigma_{0}$ in it such that

$$
M_{\Gamma}, w_{0}, \sigma_{0} \models \varphi \text { for all } \varphi \in \Gamma \text {. }
$$


Proof. If $\Gamma$ is consistent with the formula $\ell=\infty$, then we can take the model $M=$ $\langle F, \mathbf{W}, I, P\rangle$ constructed in Section 4.7 for $\Gamma \cup\{\ell=\infty\}$. Otherwise $\Gamma$ is consistent with the formula $\ell=c \wedge c<\infty$ for some rigid constant $c \notin \mathbf{L}$ and we can take $M$ from Section 4.7 for the set (4.1). In both cases $M_{\Gamma}$ can be chosen to be $\left\langle F, \mathbf{W}, \lambda w \cdot\left(\left.I^{w}\right|_{\mathbf{L}}\right), P\right\rangle$ where $\left.I^{w}\right|_{\mathbf{L}}$ stands for the restriction of $I_{w}$ to the initially given vocabulary $\mathbf{L}$, and $w_{0}$ can be chosen to be $\langle\langle\rangle, \emptyset\rangle$ where \langle\rangle is the only element of $S_{0}$ and $\emptyset$ denotes the empty function $\emptyset \rightarrow C^{d} \cup C^{p}$. In the first case the interval $\sigma_{0}$ can be chosen to be the entire time domain $T$ of $F$. In the second case $\sigma_{0}$ can be chosen to be $\left[\min T, I^{w_{0}}(c)\right]$ where $c$ is the constant introduced above. The equivalence now follows from the definition of $\Delta$ and Lemma 4.13 .

\section{Axioms For global Probability in PITL MODELS}

We call the models for PITL introduced in Definition 2.4 general, because the probability functions $\lambda X . P^{w}(\tau, X)$ in them can be arbitrary, whereas it is natural to require these functions to satisfy certain constraints. Applications typically lead to models in which all the probability functions originate from a global probability function on the entire $\mathbf{W}$ such as the automata-based models of $P D C$. Consider models $M=\langle F, \mathbf{W}, I, P\rangle$ with frames $F=\langle\langle T, \leq, \infty\rangle,\langle D,+, 0, \infty\rangle,\langle U,+, 0,1\rangle, m\rangle$ whose time domain has a least element $\tau_{0}=\min T$ and a distinguished $w_{0} \in \mathbf{W}$ such that $\mathbf{W}_{w_{0}, \tau_{0}}=\mathbf{W}$. Then $\lambda X . P^{w_{0}}\left(\tau_{0}, X\right)$ can be regarded as the global probability function and, given an arbitrary $w \in \mathbf{W}$ and $\tau \in T$, the probability function $\lambda X . P^{w}(\tau, X)$ should represent conditional probability on sets of interpretations, the condition being $\tau$-equivalence with $w$. Hence we should have

$$
P^{w_{0}}\left(\tau_{0}, \mathbf{W}_{w, \tau}\right) \cdot P^{w}(\tau, A)=P^{w_{0}}\left(\tau_{0}, \mathbf{W}_{w, \tau} \cap A\right)
$$

with respect to an appropriately defined operation of multiplication . on the probability domain for all $A \subseteq \mathbf{W}$ such that the above equality is defined. This equality is usually insufficient to determine $\lambda X . P^{w}(\tau, X)$, because, e.g., it is possible that $P^{w_{0}}\left(\tau, \mathbf{W}_{w, \tau}\right)=0$. A more general constraint of this form can be formulated as follows. Let $M, w$ and $A \subseteq \mathbf{W}$ be as above, $\tau, \tau^{\prime} \in T$ and $\tau \leq \tau^{\prime}$. Then

$$
P^{w_{0}}(\tau, A)=\int_{w \in \mathbf{W}_{w_{0}, \tau}} P^{w}\left(\tau^{\prime}, A\right) d\left(\lambda X . P^{w_{0}}(\tau, X)\right) .
$$

The integral above is not guaranteed to exist for an arbitrary probability domain, because its definition involves least upper bounds and greatest lower bounds of sets of approximating sums, which may be unavailable if there are Dedekind gaps, which is the case if, e.g., the probability domain is based the non-negative rational numbers. Dedekind-completeness is not a first-order property and therefore our proof system for PITL cannot be extended to one that is complete with respect to Dedekind-complete domains by finitary means. In this section we propose axioms which enforce the best possible approximation of (15.2) permitted by the probability domain.

In the rest of the section we consider PITL models $\langle F, \mathbf{W}, I, P\rangle$ with the probability domains of their frames $F$ extended to have multiplication. Given

$F=\langle\langle T, \leq, \infty\rangle,\langle D,+, 0, \infty\rangle,\langle U,+, ., 0,1\rangle, m\rangle$, we assume that the new operation satisfies, e.g., the following axioms: 


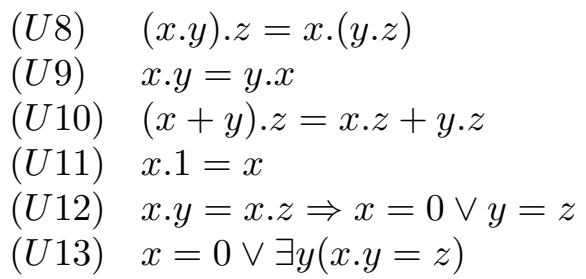

Together with $(U 1)-(U 7)$, these axioms are sufficient to extend a probability domain to a field by introducing negative elements and division in the customary way.

We adopt a definition for the integral in (5.2) which is based on Darboux-Lebesgue sums as known from the theory of integration of real-valued functions. Let the measurable sets $B_{0}, \ldots, B_{n}$ form a partition of $\mathbf{W}_{w_{0}, \tau}$ and let $P^{w}\left(\tau^{\prime}, A\right) \in\left[\xi_{i}, \eta_{i}\right]$ for all $w \in B_{i}, i=0, \ldots, n$. Then the sums

$$
\sum_{i=0}^{n} \xi_{i} P^{w_{0}}\left(\tau, B_{i}\right) \text { and } \sum_{i=0}^{n} \eta_{i} P^{w_{0}}\left(\tau, B_{i}\right)
$$

are a lower and an upper approximation for the integral from (5.2), respectively. The integral is defined if both the least upper bound of the lower approximations and the greatest lower bound of the upper approximations of the above forms taken for all partitions $B_{0}, \ldots, B_{n}$ of $\mathbf{W}_{w, \tau}$ into measurable subsets and all appropriate boundary probabilities $\xi_{i}, \eta_{i}, i=0, \ldots, n$, exist and are equal.

The sets $A$ for which $P^{w_{0}}(\tau, A)$ and $P^{w}\left(\tau^{\prime}, A\right), w \in \mathbf{W}_{w_{0}, \tau}$ need to be defined have the forms $\llbracket \varphi \rrbracket_{M, w_{0},\left[\tau^{\prime \prime}, \tau\right]}$ and $\llbracket \varphi \rrbracket_{M, w,\left[\tau^{\prime \prime}, \tau^{\prime}\right]}=\llbracket \varphi \rrbracket_{M, w_{0},\left[\tau^{\prime \prime}, \tau\right]} \cap \mathbf{W}_{w, \tau^{\prime}}$, respectively, where $\varphi$ is a formula in the vocabulary of $M$ and $\tau^{\prime \prime} \leq \tau$. Hence (5.2) can be written as

$$
P^{w_{0}}\left(\tau, \llbracket \varphi \rrbracket_{M, w_{0},\left[\tau^{\prime \prime}, \tau\right]}\right)=\int_{w \in \mathbf{W}_{w_{0}, \tau}} P\left(\tau^{\prime}, \llbracket \varphi \rrbracket_{M, w,\left[\tau^{\prime \prime}, \tau^{\prime}\right]}\right) d\left(\lambda X . P^{w_{0}}(\tau, X)\right) .
$$

Our axioms for (5.4) exploit the observation that the sets which are available for the construction of partitions $B_{0}, \ldots, B_{n}$ have such forms too. Here they are:

$(\bar{P}) \quad \ell \leq y \wedge p((\ell=y \wedge \theta \wedge p(\varphi)>x ; \top))=0 \Rightarrow p((\theta \wedge \ell=y ; \top) \wedge \varphi) \leq x \cdot p((\theta \wedge \ell=y ; \top))$ $(\underline{P}) \ell \leq y \wedge p((\ell=y \wedge \theta \wedge p(\varphi) \leq x ; \top))=0 \Rightarrow p((\theta \wedge \ell=y ; \top) \wedge \varphi) \geq x \cdot p((\theta \wedge \ell=y ; \top))$

Let us show that these axioms enforce the possible approximations of (5.4). Assume that $\bar{P}$ and $\underline{P}$ are part of our proof system. Let $\varphi$ be a PITL formula, $y$ be an individual variable of the duration sort and $x_{0}, \ldots, x_{n}$ be $n+1$ individual variables of the probability sort. Let

$$
\theta_{0} \rightleftharpoons p(\varphi) \leq x_{0}, \theta_{i} \rightleftharpoons x_{i-1}<p(\varphi) \wedge p(\varphi) \leq x_{i}, i=1, \ldots, n .
$$

Now consider the instances

$$
\ell \leq y \wedge p\left(\left(\ell=y \wedge \theta_{i} \wedge p(\varphi)>x_{i} ; \top\right)\right)=0 \Rightarrow p\left(\left(\theta_{i} \wedge \ell=y ; \top\right) \wedge \varphi\right) \leq x_{i} . p\left(\left(\theta_{i} \wedge \ell=y ; \top\right)\right)
$$

$\ell \leq y \wedge p\left(\left(\ell=y \wedge \theta_{i} \wedge p(\varphi) \leq x_{i-1} ; \top\right)\right)=0 \Rightarrow p\left(\left(\theta_{i} \wedge \ell=y ; \top\right) \wedge \varphi\right) \geq x_{i-1} \cdot p\left(\left(\theta_{i} \wedge \ell=y ; \top\right)\right)$ of $\bar{P}$ and $\underline{P}$ for $i=1, \ldots, n$ and the instance

$\ell \leq y \wedge p\left(\left(\ell=y \wedge \theta_{0} \wedge p(\varphi)>x_{0} ; \top\right)\right)=0 \Rightarrow p\left(\left(\theta_{0} \wedge \ell=y ; \top\right) \wedge \varphi\right) \leq x_{0} \cdot p\left(\left(\theta_{0} \wedge \ell=y ; \top\right)\right)$ of $\bar{P}$. Since

$$
\vdash_{P I T L} \theta_{i} \wedge p(\varphi)>x_{i} \Rightarrow \perp \text { and } \vdash_{P I T L} \theta_{i} \wedge p(\varphi) \leq x_{i-1} \Rightarrow \perp
$$

we have 


$$
\vdash_{P I T L} p\left(\left(\ell=y \wedge \theta_{i} \wedge p(\varphi)>x_{i} ; \top\right)\right)=0, p\left(\left(\ell=y \wedge \theta_{i} \wedge p(\varphi)<x_{i-1} ; \top\right)\right)=0
$$

by PITL1 and $P_{\perp}$. Hence the considered instances of $\bar{P}$ and $\underline{P}$ entail

$$
\vdash_{P I T L} \ell \leq y \Rightarrow x_{i-1} \cdot p\left(\left(\theta_{i} \wedge \ell=y ; \top\right)\right) \leq p\left(\left(\theta_{i} \wedge \ell=y ; \top\right) \wedge \varphi\right)
$$

for $i=1, \ldots, n$ and

$$
\vdash_{P I T L} \ell \leq y \Rightarrow p\left(\left(\theta_{i} \wedge \ell=y ; \top\right) \wedge \varphi\right) \leq x_{i} . p\left(\left(\theta_{i} \wedge \ell=y ; \top\right)\right)
$$

for $i=0, \ldots, n$. Let $\chi$ denote the rigid formula

$$
y<\infty \wedge x_{0}=0 \wedge x_{n}=1 \wedge \bigwedge_{i=1}^{n} x_{i-1} \leq x_{i}
$$

Then a purely ITL deduction shows that

$$
\vdash_{P I T L} \chi \Rightarrow\left(\varphi \Leftrightarrow \bigvee_{i=0}^{n}\left(\left(\theta_{i} \wedge \ell=y ; \top\right) \wedge \varphi\right)\right)
$$

and

$$
\vdash_{P I T L} \chi \Rightarrow \neg\left(\left(\left(\theta_{i} \wedge \ell=y ; \top\right) \wedge \varphi\right) \wedge\left(\left(\theta_{j} \wedge \ell=y ; \top\right) \wedge \varphi\right)\right)
$$

for $i \neq j, i, j=0, \ldots, n$. Hence, using the axioms for arithmetics of probabilities and PITL4, we can derive

$$
\vdash_{P I T L} \chi \Rightarrow p(\varphi)=\sum_{i=0}^{n} p\left(\left(\theta_{i} \wedge \ell=y ; \top\right) \wedge \varphi\right) .
$$

Now (5.5) and (5.6) imply

$$
\vdash_{P I T L} \chi \Rightarrow \sum_{i=1}^{n} x_{i-1} \cdot p\left(\left(\theta_{i} \wedge \ell=y ; \top\right)\right) \leq p(\varphi) \wedge p(\varphi) \leq \sum_{i=0}^{n} x_{i} \cdot p\left(\left(\theta_{i} \wedge \ell=y ; \top\right)\right) .
$$

Recall the model $M$ and its distinguished $w_{0} \in \mathbf{W}$ and time point $\tau_{0}$. Let $\tau, \tau^{\prime} \in T$ and $\tau \leq \tau^{\prime}$. Let $I^{w_{0}}(y)=m\left(\left[\tau_{0}, \tau^{\prime}\right]\right)$. Then the satisfaction of (5.7) at $w_{0},\left[\tau_{0}, \tau\right]$ in $M$ means that if $A=\llbracket \varphi \rrbracket_{M, w_{0}, \tau}$ and $B_{i}=\llbracket \theta_{i} \rrbracket_{M, w_{0}, \tau}, i=0, \ldots, n$, then $P^{w_{0}}(\tau, A)$ is bounded by the sums (5.3) where $\xi_{0}=0, \eta_{0}=I^{w_{0}}\left(x_{0}\right)$ and $\xi_{i}=I^{w_{0}}\left(x_{i-1}\right)$ and $\eta_{i}=I^{w_{0}}\left(x_{i}\right)$ for $i=1, \ldots, n$. Assume that $z$ is a variable of the probability sort and $M$ satisfies the rigid formula

$$
\bigwedge_{i=1}^{n} x_{i} \leq x_{i-1}+z
$$

at $w_{0}$ as well. Then, since $\sum_{i=0}^{n} P^{w_{0}}\left(\tau, B_{i}\right)=1$, the lower and upper approximations (5.3) differ by no more than $I^{w_{0}}(z)$. Now it is clear that the validity of $\bar{P}$ and $\underline{P}$ in $M$ entails that (5.4) holds approximately with precision which is smaller than any probability $\delta \in U$ such that $\underbrace{\delta+\ldots+\delta}_{n \text { times }} \geq 1$ for some $n<\omega$. Hence, if $\langle U,+, ., 0,1\rangle$ has no "infinitely small" elements, then the integral from (5.4) is defined and (5.4) holds. If there are such elements, then the difference between the least upper bound and the greatest lower bound of the sums (5.3), respectively, is "infinitely small". 
Obviously the condition $\mathbf{W}_{w_{0}, \tau_{0}}=\mathbf{W}$ is relevant just to the scope of the (approximate) validity of (5.2). If all instances of $\bar{P}$ and $\underline{P}$ hold everywhere in a PITL model, then so do the approximations of (5.2) .

\section{Probabilistic Real-time $D C$ With infinite intervals}

In this section we introduce an enhanced system of real-time probabilistic $D C$ which enables the handling of infinite intervals and has a syntactically simpler and more expressive probability operator instead of the original $\mu().($.$) . The new system is obtained as the$ extension of PITL by state expressions and duration terms. It properly subsumes the original probabilistic real-time $D C$ from [DZ99] in a straightforward way. The relative completeness result about probabilistic $D C$ in this paper is about this enhanced system and we use the acronym $P D C$ for it in the rest of the paper.

6.1. Language. $P D C$ vocabularies are just $P I T L$ vocabularies extended by state variables, which are used to construct state expressions and duration terms just like in (nonprobabilistic) $D C$ (see Section 1.2 of the Preliminaries).

6.2. Models and satisfaction. PDC models are PITL models which are based on the real-time and -probability frame for two-sorted ITL with infinite intervals

$$
F_{\mathbf{R}}=\left\langle\langle\overline{\mathbf{R}}, \leq, \infty\rangle,\left\langle\overline{\mathbf{R}}_{+},+, 0, \infty\right\rangle,\left\langle\overline{\mathbf{R}}_{+},+, ., 0,1\right\rangle, \lambda \sigma \cdot \max \sigma-\min \sigma\right\rangle
$$

the only difference being that the interpretations $I^{w}, w \in \mathbf{W}$ are supposed to map the state variables from the respective vocabularies to $\{0,1\}$-valued functions of time with the finite variability property. We assume that multiplication is available for probabilities. The definition of the values of duration terms and the definition of the satisfaction relation are just like in $D C$ and $P I T L$, respectively.

6.3. Describing probabilistic real-time automata and expressing $\mu().($.$) . The prob-$ abilistic automata from the semantics of $P D C$ originally introduced in [DZ99] can be described in the system of $P D C$ proposed in this paper. The original probability operator $\mu().($.$) can be expressed using p($.$) as follows.$

Let $\mathbf{A}$ be an automaton of the form (1.2) from Definition 1.3. The $D C$ vocabulary which corresponds to $\mathbf{A}$ consists the states of $\mathbf{A}$ as state variables and the PITL vocabulary for $\mathbf{A}$ introduced the example from Section [2.2, which includes the transitions of $\mathbf{A}$ as temporal propositional letters (0-ary flexible predicate symbols), the rigid constants $q_{a}$ and the rigid unary function symbols $P_{a}$ to denote $\lambda \tau \cdot \int_{0}^{\tau} p_{a}(t) d t$ for each transition $a$, respectively. Let $M=\left\langle F_{\mathbf{R}}, \mathbf{W}, I, P\right\rangle$ be a $P D C$ model for this vocabulary in the sense of Section 6.2 with $\mathbf{W}$ being the set of all the behaviours of $\mathbf{A}$ and $\lambda X . P^{w}(\tau, X)$ being the conditional probability for a behaviour of $\mathbf{A}$ to be described by an interpretation in the set $X \subseteq \mathbf{W}_{w, \tau}$, given that $w \in \mathbf{W}$ describes this behaviour within the interval $[0, \tau]$, like in the example from Section 2.2. Then $M$ validates the axioms

$$
\square \neg\left(\llbracket \neg a^{-} \rrbracket ; \llbracket a^{-} \rrbracket \wedge \neg a ; \llbracket a^{+} \rrbracket\right), \neg\left(\llbracket a^{-} \rrbracket \wedge \neg a ; \llbracket a^{+} \rrbracket ; \top\right)
$$

and

$$
\square\left(\neg\left(\llbracket a^{-} \rrbracket ; a\right) \wedge \neg\left(a \wedge \neg \llbracket a^{-} \rrbracket\right) \wedge \neg\left(a ; \llbracket \neg a^{+} \rrbracket\right)\right)
$$


for all transitions $a$ at all intervals $\sigma$ such that $\min \sigma=0$. These axioms force the interpretations of the temporal propositional letters $a$ to correspond to the respective transitions of $\mathbf{A}$, which are identified by observing their source states $a^{-}$and destination states $a^{+}$, in the way proposed in the example from Section 2.2. Having this correspondence, the probabilistic behaviour of $\mathbf{A}$ can be described by formulas such as (2.4). If used together with the axioms $\bar{P}$ and $\underline{P}$ from Section 5 , such formulas are sufficient to express the conditions on the probability functions $\lambda X . P^{w}(\tau, X)$ for $w \in \mathbf{W}$ which are encoded by the components $p_{a}$ and $q_{a}$ of the automaton A. Furthermore, the value of $\mu(\varphi)(t)$ is equal to $w_{[0,0]}(p((\varphi \wedge \ell=t ; \top)))$ for every $D C$ formula $\varphi$ and every $w \in \mathbf{W}$.

Note that the probabilities expressed by terms of the form $p(\varphi)$ are determined by using the truth values of $\varphi$ at infinite intervals. That is why the probability for $\varphi$ to hold at a finite interval ending at some future time point is expressed by the term $p((\varphi ; \top))$, in which $T$ accounts of the infinite interval following that end point.

In our $P D C$ axioms about probabilistic timed automata behaviour we refer to the probability $P_{a}(\tau)$ for transition $a$ to be over by time $\tau$ instead of the probability density $p_{a}(t)$ for $a$ to finish at time $t$, which was used in the original paper [DZ99]. This is not a limitation, because, at least in the case of piece-wise continuous $p_{a}$, the relation $P_{a}(\tau)=\int_{0}^{\tau} p_{a}(t) d t$ between $P_{a}$ and $p_{a}$ can be axiomatised much like (5.2). On the contrary, there are practically interesting cases such as that of transitions with discrete or finite sets of possible durations in which $p_{a}$ cannot be defined whereas $P_{a}$ exists.

\section{A PROOF SYSTEM FOR $P D C$}

The proof system for $P D C$ that we propose consists of the $D C$ axioms $D C 1-D C 6, T 1$ and $T 2$ from Section 1.2.4. We demonstrate the relative completeness of this proof system in Section 8 below. Since completeness relative to validity in the class of the PITL models which are based on $F_{\mathbf{R}}$ means that all formulas which are valid at such PITL models are admitted as axioms, the PITL axioms from Section 3 are no more relevant than any of these valid formulas from the formal point of view.

\section{Relative COMPleteness of the PROOF System for PDC}

The proof of the completeness of the axioms $D C 1-D C 6, T 1$ and $T 2$ for $P D C$ relative to validity in the class of the $F_{\mathbf{R}}$-based models of PITL follows closely the pattern of the original relative completeness proof for (non-probabilistic) $D C$ from [HZ92]. The variant of this proof about the system of $D C$ based on the modalities of $N L$ from [RZ97] is very close to our setting. Therefore we include the proof details mostly for the sake of completeness. Below PITL $\mathbf{\mathbf { R }}$ stands for the set of the PITL formulas written in the vocabulary $\mathbf{L}$ which are valid in the class of all $F_{\mathbf{R}}$-based PITL models.

Let $\varphi$ be a $P D C$ formula written in some vocabulary $\mathbf{L}$ and let $\mathbf{S}$ be the set of all the state expressions which can be written using only the state variables which occur in $\varphi$. Given a state expression $S \in \mathbf{S}$, we denote the set

$$
\left\{S^{\prime} \in \mathbf{S}: S^{\prime} \text { is propositionally equivalent to } S\right\}
$$

by $[S]$. Since $\varphi$ contains a finite number of state variables, there are finitely many different equivalence classes $[S]$ for $S \in \mathbf{S}$. Let $\mathbf{L}^{\prime}$ be the $I T L$ vocabulary which consists of the 
symbols from $\mathbf{L}$, except the state variables, and the fresh flexible constants $\ell_{[S]}, S \in \mathbf{S}$. Since there are finitely many classes $[S]$, these flexible constants are finitely many too. If all the state expressions which occur in some $P D C$ formula $\psi$ are from $\mathbf{S}$, we denote the result of substituting every duration term $\int S$ with the respective flexible constant $\ell_{[S]}$ in $\psi$ by $\psi^{\prime}$. Note that $\psi^{\prime}$ is a PITL formula with no PDC-specific constructs left in it.

Now consider the set $\mathbf{H}$ of all the instances of $D C 1-D C 6, T 1$ and $T 2$ for state expressions from $\mathbf{S}$. Unless no state variables occur in $\varphi, \mathbf{H}$ is infinite. However, since there are finitely many equivalence classes $[S]$, the set

$$
\mathbf{H}^{\prime}=\left\{\alpha^{\prime}: \alpha \in \mathbf{H}\right\}
$$

is finite. We define the sequence of formulas $\psi_{k}, k<\omega$ as follows:

$$
\psi_{0} \rightleftharpoons \square \bigwedge \mathbf{H}^{\prime}, \psi_{k+1} \rightleftharpoons \square \bigwedge \mathbf{H}^{\prime} \wedge p\left(\psi_{k}\right)=1 \text { for all } k<\omega .
$$

The formula $\psi_{k}$ states that all the instances of the $D C$ axioms hold with probability 1 at interpretations which are accessible through probability terms of height at most $k$.

Now assume that $\varphi$ is consistent with our proof system for $P D C$. Let $n=h(\varphi)$ where $h(\varphi)=0$ for $\varphi$ with no occurrence of probability terms, and $h(\varphi)=1+\max \{h(\psi)$ : $p(\psi)$ occurs in $\varphi\}$ for $\varphi$ with probability terms. Then the formula

$$
\psi \rightleftharpoons \ell=\infty \wedge\left(\varphi^{\prime} \vee\left(\varphi^{\prime} ; \ell=\infty\right)\right) \wedge \psi_{n}
$$

is consistent with $P I T L_{\mathbf{L}}^{\mathbf{R}}$. This entails that there is a $P I T L$ model $M=\left\langle F_{\mathbf{R}}, \mathbf{W}, I, P\right\rangle$, $w_{0} \in \mathbf{W}$ and an interval $\sigma_{0} \in \tilde{\mathbf{I}}(\overline{\mathbf{R}})$ such that

$$
M, w_{0}, \sigma_{0} \models \psi \text {. }
$$

Clearly $\sigma_{0} \in \mathbf{I}^{\text {inf }}(\overline{\mathbf{R}})$. Following the example from [HZ92], we use $M$ in order to build a $P D C$ model for $\mathbf{L}$ which satisfies $\varphi$.

We define the ascending sequence of subsets $\mathbf{N}_{0} \subseteq \mathbf{N}_{1} \subseteq \ldots \subseteq \mathbf{N}_{n}$ of $\mathbf{W}$ by the equalities

$$
\mathbf{N}_{0}=\left\{w_{0}\right\} \text { and } \mathbf{N}_{k}=\bigcup_{w \in \mathbf{N}_{k-1}}\left\{v \in \mathbf{W}_{w, \min \sigma_{0}}: M, v, \sigma_{0} \models \psi_{n-k}\right\} \text { for } k=1, \ldots, n .
$$

The set of the behaviour descriptions $\mathbf{W}^{\prime}$ for the $P D C$ model we are constructing is $\mathbf{N}_{n}$.

Let $w \in \mathbf{N}_{n}$ and $\tau \in\left(\min \sigma_{0}, \infty\right)$. Let $Q$ be a state variable occurring in $\varphi$. Then

$$
\ell=0 \vee(\llbracket Q \rrbracket ; \top) \vee(\llbracket \neg Q \rrbracket ; \top), \ell=0 \vee \ell=\infty \vee(\top ; \llbracket Q \rrbracket) \vee(\top ; \llbracket \neg Q \rrbracket) \in \mathbf{H},
$$

because these formulas are instances of $T 1$ and $T 2$, respectively. This entails that

$$
M, w,[\tau, \tau+1] \models\left(\ell_{[Q]}=\ell \wedge \ell \neq 0 ; \top\right) \vee\left(\ell_{[\neg Q]}=\ell \wedge \ell \neq 0 ; \top\right)
$$

and

$$
M, w,\left[\min \sigma_{0}, \tau\right] \models\left(\top ; \ell_{[Q]}=\ell \wedge \ell \neq 0\right) \vee\left(\top ; \ell_{[\neg Q]}=\ell \wedge \ell \neq 0\right),
$$

which implies that there are some $\xi, \eta \in \mathbf{R}$ such that $\xi<\tau<\eta$ and

$$
M, w,[\tau, \eta] \models \ell_{[Q]}=\ell \vee \ell_{[\neg Q]}=\ell \text { and } M, I,[\xi, \tau] \models \ell_{[Q]}=\ell \vee \ell_{[\neg Q]}=\ell .
$$

Let us fix some $\xi$ and $\eta$ with this property and denote the open neighbourhood $(\xi, \eta)$ of $\tau$ by $O_{Q, w, \tau}$. Similarly,

$$
M, w,\left[\min \sigma_{0}, \min \sigma_{0}+1\right] \models\left(\ell_{[Q]}=\ell \wedge \ell \neq 0 ; \top\right) \vee\left(\ell_{[\neg Q]}=\ell \wedge \ell \neq 0 ; \top\right)
$$


and hence there is an $\eta>\min \sigma_{0}$ such that

$$
M, w,\left[\min \sigma_{0}, \eta\right] \models \ell_{[Q]}=\ell \vee \ell_{[\neg Q]}=\ell .
$$

We fix such an $\eta$ and write $O_{Q, w, \min \sigma_{0}}$ for the semi-open neighbourhood $\left[\min \sigma_{0}, \eta\right)$ of $\min \sigma_{0}$. Obviously

$$
\bigcup_{\tau \in\left[\min \sigma_{0}, \infty\right)} O_{Q, w, \tau}=\left[\min \sigma_{0}, \infty\right)
$$

Moreover, $\mathbf{O}_{Q, w}=\left\{O_{Q, w, \tau}: \tau \in\left[\min \sigma_{0}, \infty\right)\right\}$ is a (relatively) open covering of $\left[\min \sigma_{0}, \infty\right)$. Here follows the key observation in this proof: the compactness of the intervals of the form $\left[\min \sigma_{0}+k, \min \sigma_{0}+k+1\right]$ where $k=0,1,2, \ldots$ implies that for every such $k$ there is a finite sub-covering $\mathbf{O}_{Q, w, k} \subset \mathbf{O}_{Q, w}$ of $\left[\min \sigma_{0}+k, \min \sigma_{0}+k+1\right]$. Let $\mathbf{O}_{Q, w, k}=$ $\left\{O_{Q, w, \tau_{Q, w, k, 1}}, \ldots, O_{Q, w, \tau_{Q, w, k, n}, k}\right\}$. We will use the time points $\tau_{Q, w, k, i}, i=1, \ldots, n_{w, k}$, $k=0,1, \ldots$, where $Q$ is a state variable occurring in $\varphi$ to define an interpretation $\left(I^{\prime}\right)^{w}$ of $\mathbf{L}$ in our $P D C$ model under construction which corresponds to $I^{w}$ for $w \in \mathbf{W}^{\prime}$. Let us denote the set of these time points by $C_{Q, w}$. Since $\min \sigma_{0} \in C_{Q, w}$ and $C_{Q, w} \cap \sigma$ is finite for every bounded interval $\sigma$, the set $C_{Q, w} \cap\left[\min \sigma_{0}, \tau\right]$ contains a greatest time point for every $\tau \in\left[\min \sigma_{0}, \infty\right) .\left(I^{\prime}\right)^{w}$ is defined by the following clauses

$\left(I^{\prime}\right)^{w}(s)=I(s)$ for all symbols $s \in \mathbf{L}$ which are not state variables;

$\left(I^{\prime}\right)^{w}(Q)(\tau)=0$ for all state variables $Q \in \mathbf{L}$ which do not occur in $\varphi$ and all $\tau \in \overline{\mathbf{R}}$;

$\left(I^{\prime}\right)^{w}(Q)(\tau)=1$ for state variables $P$ which occur in $\varphi$ and $\tau$ such that $M, w,\left[\tau^{\prime}, \sup O_{Q, w, \tau^{\prime}}\right] \models \ell_{[Q]}=\ell$, where $\tau^{\prime}=\max \left(C_{Q, w} \cap\left[\min \sigma_{0}, \tau\right]\right) ;$

$\left(I^{\prime}\right)^{w}(Q)(\tau)=0$ for state variables $Q$ which occur in $\varphi$ and $\tau$ such that $M, w,\left[\tau^{\prime}, \sup O_{Q, w, \tau^{\prime}}\right] \models \ell_{[\neg Q]}=\ell$, where $\tau^{\prime}$ is as above and for $\tau<\min \sigma_{0}$ as well.

A straightforward argument based on the presence of the appropriate instances of $D C 1-D C 6$ in $\mathbf{H}$ implies that this definition of $\left(I^{\prime}\right)^{w}$ is correct and $I^{\prime}$ satisfies the equality

$$
\left(I^{\prime}\right)_{\sigma}^{w}\left(\int S\right)=I_{\sigma}^{w}\left(\ell_{[S]}\right)
$$

for all state expressions $S \in \mathbf{S}$ and all intervals $\sigma \in \tilde{\mathbf{I}}(\bar{R})$ such that $\min \sigma_{0} \leq \min \sigma$.

The functions $\left(P^{\prime}\right)^{w}, w \in \mathbf{W}^{\prime}$, are defined using the respective $P^{w}$ by the equality

$$
\left(P^{\prime}\right)^{w}\left(\tau, A \cap \mathbf{W}^{\prime}\right)=P^{w}(\tau, A)
$$

for $w \in \bigcup_{i=0}^{n-1} \mathbf{N}_{i}$ and $\tau \geq \min \sigma$. Since $M, w_{0}, \sigma_{0} \models \psi_{n}$, the construction of $\mathbf{W}^{\prime}$ implies that $P^{w}\left(\tau,\left(\mathbf{W}^{\prime}\right)_{w, \tau}\right)=1$ for all such $w$. Hence if $P\left(\tau, A_{1}\right) \neq P\left(\tau, A_{2}\right)$, then $P\left(\tau, A_{1} \cap \mathbf{W}_{w, \tau}^{\prime}\right) \neq$ $P\left(\tau, A_{2} \cap \mathbf{W}_{w, \tau}^{\prime}\right)$ as well, which implies that $A_{1} \cap\left(\mathbf{W}^{\prime}\right)_{w, \tau} \neq A_{2} \cap\left(\mathbf{W}^{\prime}\right)_{w, \tau}$. That is why the equality (8.1) defines the function $\left(P^{\prime}\right)^{w}$ correctly. We allow $\left(P^{\prime}\right)^{w}$ to be arbitrary for $w \in \mathbf{W}^{\prime} \backslash \bigcup_{i=0}^{n-1} \mathbf{N}_{i}$, because the truth values of formulas of probability height up to $n$ at $w_{0}, \sigma_{0}$ do not depend on these functions.

Let $M^{\prime}=\left\langle F_{\mathbf{R}}, \mathbf{W}^{\prime}, I^{\prime}, P^{\prime}\right\rangle$. An induction on $k$ implies that if $\psi$ is a $P D C$ formula written in $\mathbf{L}, h(\psi) \leq k, w \in \mathbf{N}_{i}, \sigma \in \tilde{\mathbf{I}}(\overline{\mathbf{R}}), \min \sigma \geq \min \sigma_{0}$ and $k+i \leq n$, then

$$
M^{\prime}, w, \sigma \models \psi \text { iff } M, w, \sigma \models \psi^{\prime} \text { and } P^{w}\left(\max \sigma, \llbracket \psi^{\prime} \rrbracket_{M, w, \sigma}\right)=\left(P^{\prime}\right)^{w}\left(\tau, \llbracket \psi \rrbracket_{M^{\prime}, w, \sigma}\right) .
$$

This, in particular, implies that

$$
M^{\prime}, w_{0}, \sigma_{0} \models \varphi \text { or } M^{\prime}, w_{0}, \sigma_{0} \models(\varphi ; \ell=\infty) .
$$

In the latter case $M^{\prime}, w_{0}, \sigma \models \varphi$ for some $\sigma \in \mathbf{I}^{f i n}(\overline{\mathbf{R}})$ such that $\min \sigma=\min \sigma_{0}$. 
This concludes the proof of the relative completeness of the axioms $D C 1-D C 6, T 1$ and $T 2$ for $P D C$, because we have shown that the assumption that a given $P D C$ formula is consistent with this proof system entails that the formula is satisfiable at a $P D C$ model.

\section{PITL With Infinite InTERVAls AND $P N L$}

The system which is closest to PITL both in its semantics and proof system is the probabilistic extension of neighbourhood logic $P N L$ which was proposed in Gue00. The modalities $\diamond_{l}$ and $\diamond_{r}$ of $N L$ are defined by the clauses:

$$
\begin{aligned}
& M, \sigma \models \diamond_{l} \varphi \quad \text { iff } M, \sigma^{\prime} \models \varphi \text { for some } \sigma^{\prime} \text { such that } \max \sigma^{\prime}=\min \sigma \\
& M, \sigma \models \diamond_{r} \varphi \quad \text { iff } M, \sigma^{\prime} \models \varphi \text { for some } \sigma^{\prime} \text { such that } \min \sigma^{\prime}=\max \sigma
\end{aligned}
$$

$\diamond_{l}$ and $\diamond_{r}$ are called expanding modalities because they allow access outside the reference interval. The dual modalities $\square_{d}$ of $\diamond_{d}$ are defined by the clauses

$$
\diamond_{d} \rightleftharpoons \neg \diamond_{r} \neg \diamond_{d} \varphi
$$

for $d \in\{l, r\}$.

A duration calculus on the basis of $N L$ was developed in [RZ97]. Infinite intervals are an alternative way to achieve the expressivity of $\diamond_{r}$. A truth preserving translation from $I T L$ with infinite intervals to $N L$ is impossible for the trivial reason that $N L$ does not have infinite intervals and there is no straightforward way to capture the ITL interpretation of flexible symbols at infinite intervals. Furthermore, $N L$ duration domains known from the literature do not include $\infty$, but include negative durations. However, if the only flexible symbols in the considered vocabularies are $\ell$ and state variables, then the duration calculi based on $N L$ and on ITL with infinite intervals, respectively, can be related by means of a translation which has the following property:

If $\psi$ is the $N L$-based $D C$ formula which is the translation of some $I T L$-based

$D C$ formula $\varphi$ and $F V(\varphi)=\left\{x_{1}, \ldots, x_{n}\right\}$, then

$$
M^{\prime},[\tau, \tau] \models \psi \text { iff } M,[\tau, \infty] \models \varphi,
$$

where the duration domain of the ITL model $M$ is obtained from that of the $N L$ model $M^{\prime}$ by removing the negative elements and adding $\infty$, and the meanings of the non-logical symbols in $M$ and $M^{\prime}$ on the intersection of the two duration domains are the same. We describe such a translation in this section.

The predicate logic equivalences

$$
R\left(t_{1}, \ldots, t_{n}\right) \Leftrightarrow \exists x_{1} \ldots \exists x_{n}\left(R\left(x_{1}, \ldots, x_{n}\right) \wedge \bigwedge_{i=1}^{n} t_{i}=x_{i}\right)
$$

and

$$
f\left(t_{1}, \ldots, t_{n}\right)=z \Leftrightarrow \exists x_{1} \ldots \exists x_{n}\left(f\left(x_{1}, \ldots, x_{n}\right)=z \wedge \bigwedge_{i=1}^{n} t_{i}=x_{i}\right),
$$

where $x_{1}, \ldots, x_{n}$ do not occur in $t_{1}, \ldots, t_{n}$, allow us to assume that all atomic subformulas of the $I T L$ formulas to be translated are either rigid of have the form $\int S=x$ where $x$ is a variable. We can also treat $\ell$ as $\int \mathbf{1}$. The clauses below define two auxiliary translations (.) $)^{f i n}$ and (.) $)^{\text {inf }}$ from ITL-based to $N L$-based $D C$. (.) $)^{\text {fin }}$ translates an ITL formula which is to be evaluated at a finite interval into its $N L$ equivalent. (. $)^{\text {inf }}$ translates an ITL formula which is to be evaluated at an infinite interval $\sigma$ into a corresponding $N L$ formula which 
defines the same condition on $\sigma$ when evaluated at the zero-length interval $[\min \sigma, \min \sigma]$. $(.)^{\text {inf }}$ refers to $(.)^{f i n}$ for the translation of (.;.)-formulas. Both auxiliary translations are correct only under the assumption that the free variables of the given ITL formulas range over non-negative finite durations. Infinity is handled only where explicitly denoted by the symbol $\infty$. Atomic formulas $R\left(t_{1}, \ldots, t_{n}\right)$ with the parameter list $t_{1}, \ldots, t_{n}$ consisting of individual variables and, possibly, $\infty$ translate into dedicated specialising formulas $S_{t_{1}, \ldots, t_{n}}^{R}$, which define the appropriate predicates on the non- $\infty$ parameters according to the intended meaning of $R$ and the positions of the occurrences of $\infty$ in $t_{1}, \ldots, t_{n}$. For instance, $S_{x, y}^{=}$ is $x=y, S_{x, \infty}^{=}$is $\perp$, and $S_{\infty, \infty}^{=}$is $\top$. Atomic formulas with $=$ and function symbols are handled similarly, e.g. the formula $S_{x, \infty ; y}^{+}$for $x+\infty=y$ is $\perp$, and $S_{x, \infty ; \infty}^{+}$is $\top$.

$$
\begin{aligned}
& \perp^{\text {fin }} \quad \rightleftharpoons \perp \\
& \left(R\left(t_{1}, \ldots, t_{n}\right)\right)^{f i n} \quad \rightleftharpoons S_{t_{1}, \ldots, t_{n}}^{R} \\
& \left(f\left(t_{1}, \ldots, t_{n}\right)=t_{n+1}\right)^{f i n} \rightleftharpoons S_{t_{1}, \ldots, t_{n} ; t_{n+1}}^{f} \\
& \left(\int S=\infty\right)^{\text {fin }} \quad \rightleftharpoons \perp \\
& \left(\int S=x\right)^{\text {fin }} \quad \rightleftharpoons \int S=x \\
& (\varphi \Rightarrow \psi)^{\text {fin }} \quad \rightleftharpoons \varphi^{\text {fin }} \Rightarrow \psi^{\text {fin }} \\
& (\varphi ; \psi)^{\text {fin }} \quad \rightleftharpoons \exists x \exists y\left(\int \mathbf{1}=x+y \wedge \diamond_{l} \diamond_{r}\left(\ell=x \wedge \varphi^{\text {fin }} \wedge \diamond_{r}\left(\ell=y \wedge \psi^{f i n}\right)\right)\right) \\
& (\exists x \varphi)^{f i n} \quad \rightleftharpoons([\infty / x] \varphi)^{f i n} \vee \exists x\left(x \geq 0 \wedge \varphi^{f i n}\right)
\end{aligned}
$$

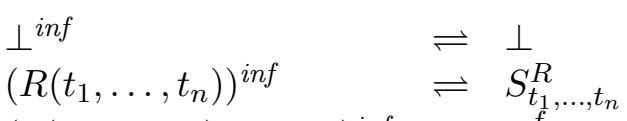

$$
\begin{aligned}
& \left(f\left(t_{1}, \ldots, t_{n}\right)=t_{n+1}\right)^{i n f} \rightleftharpoons S_{t_{1}, \ldots, t_{n} ; t_{n+1}}^{f} \\
& \left(\int S=\infty\right)^{i n f} \quad \rightleftharpoons \forall x \diamond_{r} \int S>x \\
& \left(\int S=x\right)^{i n f} \quad \rightleftharpoons \diamond_{r}\left(\int S=x \wedge \square_{r} \int S=0\right) \\
& (\varphi \Rightarrow \psi)^{i n f} \quad \rightleftharpoons \varphi^{i n f} \Rightarrow \psi^{i n f} \\
& (\varphi ; \psi)^{i n f} \quad \rightleftharpoons \diamond_{r}\left(\varphi^{\text {fin }} \wedge \diamond_{r}\left(\ell=0 \wedge \psi^{\text {inf }}\right)\right) \\
& (\exists x \varphi)^{\text {inf }} \quad \rightleftharpoons([\infty / x] \varphi)^{\text {inf }} \vee \exists x\left(x \geq 0 \wedge \varphi^{\text {inf }}\right)
\end{aligned}
$$

As mentioned above, (. $)^{\text {inf }}$ is correct only under the assumption that the free variables of the given ITL formulas range over non-negative finite durations. To remove this restriction, given an ITL formula $\varphi$ whose free variables are $x_{1}, \ldots, x_{n}$, we define the sequence of formulas $\varphi_{0}, \ldots, \varphi_{n}$ by the clauses

$$
\varphi_{0} \rightleftharpoons \varphi \text { and } \varphi_{i} \rightleftharpoons\left(x_{i} \geq 0 \wedge \varphi_{i-1}\right) \vee\left[\infty / x_{i}\right] \varphi_{i-1} \text { for } i=1, \ldots, n,
$$

and choose the formula $\psi$ from (9.1) to be $\left(\varphi_{n}\right)^{i n f}$. This translation can be extended to one between $P D C$ with infinite intervals and a system of probabilistic $D C$ based on $N L$ by putting

$$
\begin{aligned}
& (p(\varphi)=x)^{\text {fin }} \rightleftharpoons p\left(\varphi^{\text {inf }}\right)=x \\
& (p(\varphi)=x)^{\text {inf }} \rightleftharpoons \varphi^{\text {inf }} \wedge x=1 \vee \neg \varphi^{\text {inf }} \wedge x=0 .
\end{aligned}
$$

A translation from $N L$ into $I T L$ with infinite intervals is possible too under the assumption that there is a time point $\tau_{0}$ such that the values of all flexible symbols except $\ell$ at intervals starting before $\tau_{0}$ are irrelevant to the truth value of the translated formula. This restriction is necessary, because an ITL formula cannot express conditions on the past prior to the beginning of the infinite reference interval. It can be avoided if one considers a system of ITL with intervals which can be infinite into the past as well, which is beyond the scope of 
this paper. If a property does not depend on the interpretation of the flexible symbols on the left of the beginning of the reference interval and can be expressed by an $N L$ formula, then it can be expressed by an $N L$ formula in which the only occurrences of $\diamond_{l}$ are in subformulas of the form $\diamond_{l} \diamond_{r} \chi$. Given an $N L$ formula $\varphi$ which satisfies this syntactical restriction, one can find an ITL formula $\psi$ such that $M,\left[\tau_{0}, \infty\right] \models \psi$ is equivalent to the existence of a $\tau_{1} \geq \tau_{0}$ such that $M^{\prime},\left[\tau_{0}, \tau_{1}\right] \models \varphi$. Below we give a translation which, given a $\varphi$ of the form

$$
\varphi::=\perp|R(t, \ldots, t)|(\varphi \Rightarrow \varphi)\left|\diamond_{r} \varphi\right| \diamond_{l} \diamond_{r} \varphi \mid \exists x(x \geq 0 \wedge \varphi)
$$

produces a corresponding $\psi$. This translation produces formulas constructed using $\exists, \Rightarrow$, $\perp$, rigid formulas and formulas of the form

$$
\left(\ell=t_{1} ; \ell=t_{2} \wedge \alpha ; \top\right)
$$

with $\alpha$ being a modality-free formula. The translation works by reducing the number of the occurrences of $\diamond_{l} \diamond_{r}$ and $\diamond_{r}$ in formulas of the form (9.2), yet with $\alpha$ being a $N L$ formula. The $I T L$ formula $\psi$ is obtained by starting from $(\ell=0 ; \ell=0 \wedge \diamond \varphi ; \top)$. To understand the correctness of the translation, one can think of a system which has all the modalities (.;.), $\diamond_{l}$ and $\diamond_{r}$, with the obvious semantics, and check that the translation rules correspond to valid equivalences at infinite reference intervals, provided that the free variables of the involved formulas have finite non-negative values. Here follow the transformation rules which define the translation:

$$
\begin{aligned}
& \left(\ell=t_{1} ; \ell=t_{2} \wedge\left(\chi_{1} \Rightarrow \chi_{2}\right) ; \top\right) \rightarrow\left(\ell=t_{1} ; \ell=t_{2} \wedge \chi_{1} ; \top\right) \Rightarrow\left(\ell=t_{1} ; \ell=t_{2} \wedge \chi_{2} ; \top\right) \\
& \left(\ell=t_{1} ; \ell=t_{2} \wedge \diamond_{r} \chi ; \top\right) \rightarrow \exists z\left(\ell=t_{1}+t_{2} ; \ell=z \wedge \chi ; \top\right) \\
& \left(\ell=t_{1} ; \ell=t_{2} \wedge \diamond_{l} \diamond_{r} \chi ; \top\right) \rightarrow \exists z\left(\ell=t_{1} ; \ell=z \wedge \chi ; \top\right) \\
& \left(\ell=t_{1} ; \ell=t_{2} \wedge \exists x(x \geq 0 \wedge \chi) ; \top\right) \rightarrow \exists x\left(x<\infty \wedge\left(\ell=t_{1} ; \ell=t_{2} \wedge \chi ; \top\right)\right)
\end{aligned}
$$

The individual variable $z$ in the rules above is supposed to be fresh. The last rule can be applied only if $x \notin F V\left(t_{1}\right), F V\left(t_{2}\right)$. This translation can be extended to one from $P N L$ to PITL by mapping $N L$ probability terms $p(\varphi)$ to PITL corresponding probability terms $p(\psi)$ where $\psi$ is the translation of $\varphi$.

\section{CONCLUding REMARKS}

We conclude by discussing some restrictions on the scope of the completeness results about PITL and PDC presented in this paper.

Countable additivity of probability functions. According to our definition, the probability functions in PITL models are required to be just finitely additive, whereas classical probability theory is about countably additive probability functions. One simple reason for this is the choice to have an abstract domain of probabilities which is not required to be Dedekind-complete and therefore the infinite sums which are relevant to countable additivity cannot be guaranteed to exist. The difficulty in axiomatising countable additivity becomes even more obvious from the observation that PITL has the Löwenheim-Skolem property. This means that countably-infinite consistent sets of PITL formulas can be satisfied at countably-infinite models, which, in particular, have countably-infinite domains. This follows immediately from the construction of the PITL model in the completeness 
argument for our proof system. Countably-infinite PITL models with countably additive probability functions validate formulas of the form

$$
\forall x(p(\varphi)=0) \Rightarrow p(\exists x \varphi)=0 .
$$

This follows immediately from the fact that $x$ ranges over a countably-infinite domain. Hence, the above formula should be a theorem in a proof system which is complete with respect to models with countably additive probability functions, as long as the LöwenheimSkolem property holds. However, this formula is not valid in arbitrary models.

Completeness of PDC relative to (non-probabilistic) real-time ITL. Our demonstration that some well-known axioms of (non-probabilistic) $D C$ form a proof system which is complete relative to probabilistic ITL with infinite intervals was hardly a technical challenge, given the similar proofs from [HZ92, RZ97. It would have been interesting to develop a proof system for $P D C$ which is complete relative to real-time $I T L$ without probabilities. The proof of Lemma 4.11, which is the key step in our model construction for the completeness argument for PITL, explains why this is impossible. The model construction involves an expression of $\tau$-equivalence by the formulas

$$
\left(\square \forall\left(\chi^{\nu} \Leftrightarrow \chi^{\nu^{\prime}}\right) \wedge \ell=c ; \ell=\infty\right)
$$

for $\tau$ being the equivalence class $[c]$ of the rigid constant $c$. The relation of $\tau$-equivalence is needed to hold between any given $w \in \mathbf{W}$ from a $P D C$ model $M=\left\langle F_{\mathbf{R}}, \mathbf{W}, I, P\right\rangle$ and the $v \in \mathbf{W}$ which are needed to populate $\llbracket \varphi \rrbracket_{M, w, \sigma}$ for $\varphi$ such that $M, w$ is supposed to satisfy $p(\varphi) \neq 0$ at intervals $\sigma$ whose end point is $\tau$. The proof of Lemma 4.11 relies on the possibility to use the formulas (9.3) and an assumption which essentially amounts to the derivability of $\neg \varphi$ from some appropriately chosen formulas in order to derive the existence of a formula $\theta$ such that the same formulas imply $(\theta \wedge \ell=c ; \ell=\infty) \Rightarrow \neg \varphi$, which in its turn enables an application of the PITL proof rule $P_{\leq}$to derive $\theta \Rightarrow p(\varphi)=0$ and reach the aimed contradiction. The existence of the formula $\theta$ amounts to the interval-related intepolation property of ITL with infinite intervals (see Section 4.1). Unfortunately, $D C$ has neither this interpolation property, nor the related Craig interpolation property Gue04b]. The counterexample to Craig interpolation in Gue04b] indicates that the property could possibly be restored by allowing infinitary formulas to take the role of $\theta$. DC is not a compact logic and therefore derivability from infinite sets of premises is not reducible to derivability from finite ones. Hence, in order to achieve sufficient deductive power, the proof rule $P_{\leq}$would have to be replaced by one allowing infinitary formulas on the left of $\Rightarrow$ as well. The deductive power of a finitary rule would be insufficient for the role of $P_{\leq}$in any presumable finitary proof system for $P D C$ that is complete relative to (non-probabilistic) real-time $I T L$ with infinite intervals.

\section{REFERENCES}

[ACD91] Rajeev Alur, Costas Courcoubetis, and David L. Dill. Model-checking for Probabilistic Real-time Systems. In Proceedings of ICALP'91, volume 510 of $L N C S$, pages 115-136. Springer, 1991.

[ACD92] Rajeev Alur, Costas Courcoubetis, and David L. Dill. Verifying Automata Specifications of Probabilistic Real-time Systems. In Real-Time: Theory and Practice, volume 600 of LNCS, pages 28-44. Springer, 1992.

[ASB95] Adnan Aziz, Vigyan Singhal, and Felice Balarin. It Usually Works: The Temporal Logic of Stochastic Systems. In Proceedings of CAV'95, volume 939 of LNCS, pages 155-165. Springer, 1995. 
[BM05] Davide Bresolin and Angelo Montanari. A Tableau-based Decision Procedure for Branching Time Interval Temporal Logic. In TABLEAUX 2005. 14th Conference on Automatic Reasoning with Analytic Tableaux and Related Methods, volume 3702 of LNAI, pages 63-77. Springer, 2005.

[BMS07] Davide Bresolin, Angelo Montanari, and Pietro Sala. An Optimal Tableau-based Decision Procedure for Propositional Neighbourhood Logic. In STACS 200\%. 24th International Symposium on Theoretical Aspects of Computer Science, 2007. to appear.

[BRZ00] Rana Barua, Suman Roy, and Zhou Chaochen. Completeness of Neighbourhood Logic. Journal of Logic and Computation, 10(2):271-295, 2000.

[CK73] C. C. Chang and H. J. Keisler. Model Theory. North Holland, 1973. The book has had more recent editions.

[Dan98] Dang Van Hung. Modelling and Verification of Biphase Mark Protocols in Duration Calculus Using PVS/DC ${ }^{-}$. In Proceedings of the 1998 International Conference on Application of Concurrency to System Design (CSD'98), pages 88-98. IEEE Computer Society Press, March 1998.

[Dut95a] Bruno Dutertre. On First-order Interval Temporal Logic. Report CSD-TR-94-3, Department of Computer Science, Royal Holloway, University of London, Egham, Surrey TW20 0EX, England, 1995. A short version appeared as Dut95b.

[Dut95b] Bruno Dutertre. On First Order Interval Temporal Logic. In Proceedings of LICS'95, pages 36-43. IEEE Computer Society Press, 1995.

[DW96] Dang Van Hung and Wang Ji. On The Design of Hybrid Control Systems Using Automata Models. In Proceedings of FST TCS 1996, volume 1180 of LNCS, pages 156-167. Springer, 1996.

[DZ99] Dang Van Hung and Zhou Chaochen. Probabilistic Duration Calculus for Continuous Time. Formal Aspects of Computing, 11(1):21-44, 1999.

[Gue98] Dimitar P. Guelev. Probabilistic Interval Temporal Logic. Technical Report 144, UNU/IIST, P.O.Box 3058, August 1998. Draft.

[Gue00] Dimitar P. Guelev. Probabilistic Neighbourhood Logic. In Mathai Joseph, editor, Proceedings of FTRTFT 2000, volume 1926, pages 264-275. Springer, 2000. A proof-complete version is available as UNU/IIST Technical Report 196 from http://www.iist.unu.edu.

[Gue01] Dimitar P. Guelev. Interval-related Interpolation in Interval Temporal Logics. Logic Journal of the IGPL, 9(5):677-685, 2001. Presented at ICTL 2000, Leipzig, October, 2000.

[Gue04a] Dimitar P. Guelev. A Complete Proof System for First-order Interval Temporal Logic with Projection. Journal of Logic and Computation, 14(2):215-249, 2004.

[Gue04b] Dimitar P. Guelev. Logical Interpolation and Projection onto State in the Duration Calculus. Journal of Applied Non-classical Logics, Special Issue on Interval Temporal Logics and Duration Calculi, 14(1-2):185-213, 2004. Presented at the ESSLLI Workshop on Interval Temporal Logics and Duration Calculi, Vienna, August, 2003.

[Gue04c] Dimitar P. Guelev. Sharpening the Incompleteness of the Duration Calculus. In Irek Ulidowski, editor, Proceedings of ARTS 2004, volume? of ENTCS. Elsevier Science, 2004. Presented at ARTS 2004, Stirling, UK.

[He 99a] He Jifeng. A Behavioral Model for Co-design. In Proceedings of FM'99, volume 1709 of LNCS, pages 1420-1438. Springer, 1999.

[He 99b] He Jifeng. Integrating Variants of DC. Research Report 172, UNU/IIST, P.O.Box 3058, Macau, August 1999.

[HS86] J. Y. Halpern and Y. Shoham. A Propositional Logic of Time Intervals. In Proceedings of LICS'86, pages 279-292. IEEE Computer Society Press, 1986.

[Hu 99] Hu Chengjun. Proof Techniques and Tools for Interval Logics. Ph.D. thesis, Changsha Institute of Technology, Changsha, China, 1999. (In Chinese).

[HZ92] Michael R. Hansen and Zhou Chaochen. Semantics and Completeness of Duration Calculus. In Real-Time: Theory and Practice, volume 600 of LNCS, pages 209-225. Springer, 1992.

[HZ97] Michael R. Hansen and Zhou Chaochen. Duration Calculus: Logical Foundations. Formal Aspects of Computing, 9:283-330, 1997.

[IEE95] IEEE Computer Society. IEEE Standard Hardware Description Language Based on the Verilog Hardware Description Language (IEEE std 1364-1995). IEEE Computer Society Press, 1995.

[Jos95] Mathai Joseph. Real-Time Systems. Prentice Hall, 1995.

[KNP01] Marta Kwiatkowska, Gethin Norman, and David Parker. PRISM: Probabilistic symbolic model checker. In P. Kemper, editor, Proc. Tools Session of Aachen 2001 International Multiconference 
on Measurement, Modelling and Evaluation of Computer-Communication Systems, pages 7-12, 2001. Available as Technical Report 760/2001, University of Dortmund.

[LH99] Li Li and He Jifeng. A Denotational Semantics of Timed RSL using Duration Calculus. In Proceedings of RTCSA'99, pages 492-503. IEEE Computer Society Press, 1999.

[LRSZ93] Liu Zhiming, A. P. Ravn, E. V. Sørensen, and Zhou Chaochen. A Probabilistic Duration Calculus. In H. Kopetz and Y. Kakuda, editors, Dependable Computing and Fault-tolerant Systems Vol. 7: Responsive Computer Systems, pages 30-52. Springer, 1993.

[McM] Ken McMillan. SMV documentation postscript versions. URL: http://www-cad.eecs.berkeley.edu/ ${ }^{\sim}$ kenmcmil/psdoc.html. Accessed in February, 2002.

[MO99] Markus Müller-Olm. A modal fixpoint logic with chop. In Proceedings of STACS'99, volume 1563 of $L N C S$, pages 510-512. Springer, 1999.

[Mon] The MONA Project. URL: http://www.brics.dk/ mona/. Maintained by Anders Mфller.

[Mos85] Ben Moszkowski. Temporal Logic For Multilevel Reasoning About Hardware. IEEE Computer, 18(2):10-19, 1985.

[Mos86] Ben Moszkowski. Executing Temporal Logic Programs. Cambridge University Press, 1986.

[Pan] Paritosh K. Pandya. DCVALID. A tool for modelchecking Duration Calculus Formulae. URL: http://www.tcs.tifr.res.in/ pandya/dcvalid.html.

[Pan95] Paritosh K. Pandya. Some extensions to Mean-Value Calculus: Expressiveness and Decidability. In Proceedings of CSL'95, volume 1092 of LNCS, pages 434-451. Springer, 1995.

[Pan96] Paritosh K. Pandya. Weak Chop Inverses and Liveness in Mean-Value Calculus. In Proceedings of FTRTFT'96, volume 1135 of LNCS, pages 148-167. Springer, 1996.

[Pan01] Paritosh K. Pandya. Model checking CTL[DC]. In Proceedings of TACAS 2001, volume 2031 of LNCS, pages 559-573. Springer, 2001.

[Pan02] Paritosh K. Pandya. The saga of synchronous bus arbiter: On model checking quantitative timing properties of synchronous programs. In Proceedings of SLAP'02, volume 65(5) of ENTCS. Elsevier Science, 2002.

[PD98] Paritosh K. Pandya and Dang Van Hung. Duration Calculus of Weakly Monotonic Time. In Proceedings of FTRTFT'98, volume 1486 of LNCS, pages 55-64. Springer, 1998.

[PRI] PRISM: Probabilistic Symbolic Model Checker. URL: http://www.cs.bham.ac.uk/ dxp/prism/. Maintained by David Parker.

[PVS] PVS Specification and Verification System. URL: http://pvs.csl.sri.com. Maintained by Sam Owre.

[PWX98] Paritosh K. Pandya, Wang Hanping, and Xu Qiwen. Towards a Theory of Sequential Hybrid Programs. In D. Gries and W.-P. de Roever, editors, Proceedings of IFIP Working Conference PROCOMET'98, pages 336-384. Chapman \& Hall, 1998.

[Rab98] Alexander Rabinovich. Non-elementary Lower Bound for Propositional Duration Calculus. Information Processing Letters, 66:7-11, 1998.

[Ras02] Thomas M. Rasmussen. Interval Logic - Proof Theory and Theorem Proving. Ph.D. thesis, Technical University of Denmark, 2002.

[RZ97] Suman Roy and Zhou Chaochen. Notes on Neighbourhood Logic. Technical Report 97, UNU/IIST, P.O.Box 3058, February 1997.

[SS94] J.U. Skakkebæk and N. Shankar. Towards a Duration Calculus Proof Assistant in PVS. In Proceedings of FTRTFT'94, volume 863 of LNCS, pages 660-679. Springer, 1994.

[SX98] Gerardo Schneider and Xu Qiwen. Towards a Formal Semantics of Verilog Using Duration Calculus. In Anders P. Ravn and Hans Rischel, editors, Proceedings of FTRTFT'98, volume 1486 of LNCS, pages 282-293. Springer, 1998.

[Tri99] Vladimir T. Trifonov. A completeness theorem for the probabilistic interval temporal logic with respect to its standard semantics. M.Sc. Thesis, Sofia University, July 1999. (In Bulgarian).

[UPP] UPPAAL. URL: http://www.uppaal.com.

[Ven91a] Yde Venema. A Modal Logic for Chopping Intervals. Journal of Logic and Computation, 1(4):453476, 1991.

[Ven91b] Yde Venema. Many-Dimensional Modal Logics. Ph.D. thesis, University of Amsterdam, 1991.

[WX04] Wang Hanpin and Xu Qiwen. Completeness of Temporal Logics over Infinite Intervals. Discrete Applied Mathematics, 136(1):87-103, 2004. 
[ZDL95] Zhou Chaochen, Dang Van Hung, and Li Xiaoshan. A Duration Calculus with Infinite Intervals. In Horst Reichel, editor, Fundamentals of Computation Theory, volume 965 of LNCS, pages 16-41. Springer, 1995.

[ZH98] Zhou Chaochen and Michael R. Hansen. An Adequate First Order Interval Logic. In International Symposium, Compositionality - The Significant Difference, volume 1536 of LNCS, pages 584-608. Springer, 1998.

[ZH04] Zhou Chaochen and Michael R. Hansen. Duration Calculus. A Formal Approach to Real-Time Systems. Springer, 2004.

[ZHR91] Zhou Chaochen, C. A. R. Hoare, and Anders P. Ravn. A Calculus of Durations. Information Processing Letters, 40(5):269-276, 1991.

[ZHS93] Zhou Chaochen, Michael R. Hansen, and P. Sestoft. Decidability and Undecidability Results for Duration Calculus. In Proceedings of STACS'93, volume 665 of LNCS, pages 58-68. Springer, 1993.

[ZZ94] Zheng Yuhua and Zhou Chaochen. A Formal Proof of a Deadline Driven Scheduler. In Proceedings of FTRTFT'94, volume 863 of LNCS, pages 756-775. Springer, 1994. 